\title{
Relative Prenatal and Postnatal Maternal Contributions to Schizophrenia-Related Neurochemical Dysfunction after In Utero Immune Challenge
}

\author{
Urs Meyer ${ }^{1,2}$, Myriel Nyffeler,2, Severin Schwendener', Irene Knuesel ${ }^{1,3}$, Benjamin K Yee*,' and \\ Joram Feldon' \\ 'Laboratory of Behavioural Neurobiology, ETH Zurich, Schwerzenbach, Switzerland
}

\begin{abstract}
Prenatal exposure to infections represents a risk factor for the emergence of neuropsychiatric disorders in later life, including schizophrenia and autism. However, it remains essentially unknown whether this association is primarily attributable to prenatal and/or postnatal maternal effects on the offspring. Here, we addressed this issue by dissecting the relative contributions of prenatal inflammatory events and postnatal maternal factors in an animal model of prenatal viral-like infection. Pregnant mice were exposed to the inflammatory agent polyriboinosinic-polyribocytidilic acid (Polyl:C; $5 \mathrm{mg} / \mathrm{kg}$, i.v.) or vehicle treatment on gestation day 9, and offspring born to Polyl:Cand vehicle-treated dams were cross fostered to surrogate rearing mothers that had either experienced inflammatory or sham treatment during pregnancy. We demonstrate that a variety of dopamine- and glutamate-related pharmacological and neuroanatomical disturbances emerge after prenatal immune challenge regardless of whether neonates were raised by vehicle- or Polyl:C-exposed surrogate mothers. However, the adoption of prenatal control animals to immune-challenged surrogate mothers was also sufficient to induce specific pharmacological and neuroanatomical abnormalities in the fostered offspring. Multiple schizophrenia-related dysfunctions emerging after prenatal immune challenge are thus mediated by prenatal but not postnatal maternal effects on the offspring, but immunological stress during pregnancy may affect postpartum maternal factors in such a way that being reared by an immune-challenged surrogate mother can confer risk for distinct forms of psychopathology in adult life.

Neuropsychopharmacology (2008) 33, 44I-456; doi: I0.1038/sj.npp. I3014I3; published online I8 April 2007
\end{abstract}

Keywords: adoption; dopamine; glutamate; infection; neurodevelopment; pregnancy

\section{INTRODUCTION}

Maternal infection during pregnancy increases the risk for several neuropsychiatric disorders in the offspring, including schizophrenia (Brown and Susser, 2002; Fatemi, 2005) and autism (Rodier and Hyman, 1998; Miller et al, 2005). Recent attempts to model this epidemiological link in animals has yielded considerable support for a causal relationship between in utero immune activation and the emergence of adult psychopathology. Structural and functional abnormalities implicated in the endophenotypes of schizophrenia and related disorders have been detected in rats and mice following prenatal exposure to various

\footnotetext{
*Correspondence: Dr BK Yee, Laboratory of Behavioural Neurobiology, ETH Zurich, Schorenstrasse 16, CH-8603 Schwerzenbach, Switzerland, Tel: +4I 44655 74I7, Fax: +4I | 655 7203,

E-mail: byee@ethz.ch

${ }^{2}$ These authors contributed equally to this work.

${ }^{3}$ Current address: Institute of Pharmacology and Toxicology, University of Zurich, Winterthurerstrasse 190, CH 8057 Zurich, Switzerland. Received 27 December 2006; revised 2 February 2007; accepted 5 March 2007
}

immunostimulatory agents, including bacterial endotoxin, human influenza virus, or a synthetic viral mimic (reviewed in Meyer et al, 2005, 2007; Nawa and Takei, 2006; Sullivan et al, 2006). Some of the behavioral deficits are responsive to known antipsychotic drugs, and this further strengthened the predictive validity of these models for schizophreniarelated psychopathology (eg Borrell et al, 2002; Zuckerman et al, 2003; Ozawa et al, 2006).

Infection-induced elevation of pro-inflammatory cytokines in the maternal host may be one of the key events leading to enhanced risk for neuropsychiatric disorders in the offspring (Gilmore and Jarskog, 1997). Support for this hypothesis is manifold. First, increased maternal levels of the pro-inflammatory cytokine tumor necrosis factor- $\alpha$ and the chemokine interleukin-8 during pregnancy have been directly associated with a higher risk for schizophrenia in the progeny (Buka et al, 2001; Brown et al, 2004). Second, experimentation in animals has confirmed that, in the absence of a specific pathogen, prenatal exposure to cytokine-releasing agents (Shi et al, 2003; Zuckerman et al, 2003; Zuckerman and Weiner, 2005; Meyer et al, 2005, 2006a-c; Ozawa et al, 2006) is sufficient to induce 
psychopathology in later life. Third, in addition to their immunological roles (Curfs et al, 1997), pro-inflammatory cytokines have various neurodevelopmental effects (eg Ling et al, 1998; Jarskog et al, 1997; Gilmore et al, 2004). Hence, an imbalance in pro-inflammatory cytokine may trigger mal-neurodevelopment in the developing fetus and ultimately precipitate neuropathology and psychopathology in the adult offspring.

In contrast to the possible events taking place at the maternal-fetal interface, the putative roles of postnatal maternal factors have received scant attention in the elucidation of the prenatal infectious etiologies of schizophrenia and related disorders (Meyer et al, 2006c). However, immunological stimulation is strongly linked to the activation of several stress response systems, including the hypothalamic-pituitary-adrenal axis (Haddad et al, 2002; Webster and Sternberg 2004); and maternal physiological and/or psychological stress during pregnancy is known to alter postpartum maternal behavior (Meek et al, 2001; Patin et al, 2002). Disruption of the intricate motherinfant relationship resulting from immunological stress during pregnancy may thus confer additional risk for the offspring to develop psychopathology in later life. We have recently found experimental evidence for this possibility by showing that the adoption of prenatal control mice to surrogate rearing mothers, which had been exposed to immunological stress during early/mid pregnancy (gestation day (GD) 9), is sufficient to induce learning disabilities in the fostered offspring (Meyer et al, 2006c).

Here, we dissected the relative contributions of prenatal and postnatal maternal factors to the emergence of dopamine- and glutamate-related pharmacological and neurochemical imbalances after prenatal immune challenge. Pregnant mice were exposed to the inflammatory agent polyriboinosinic-polyribocytidilic acid (PolyI:C) or vehicle treatment in early/mid pregnancy (GD 9), and offspring born to PolyI:C- and vehicle-treated dams were cross fostered to surrogate rearing mothers that had either experienced inflammatory or sham treatment during pregnancy. First, we evaluated the efficacy of the prenatal and postnatal manipulations to increase the locomotor reaction to systemic administration with the indirect dopamine-receptor agonists, amphetamine (AMPH), and the noncompetitive $\mathrm{N}$-methyl-D-aspartate (NMDA)-receptor antagonist, dizocilpine (MK-801), in pre-adolescent and adult offspring. Both dopamine-receptor agonists and NMDA-receptor antagonist can produce psychosis-like states in healthy human subjects and exacerbate existing psychoses in patients with schizophrenia (Lieberman et al, 1987; Javitt and Zukin, 1991; Laruelle et al, 1996). There is also experimental evidence that maternal immune activation during pregnancy enhances the offspring's sensitivity to these drugs in adulthood (Zuckerman et al, 2003; Zuckerman and Weiner, 2005; Fortier et al, 2004a; Meyer et al, 2005; Ozawa et al, 2006), but it is essentially unknown whether these pharmacological dysfunctions are attributable to prenatal and/or postnatal maternal effects on the offspring. Second, we aimed at identifying possible concomitant changes in the expression of dopamine- and glutamate-related neurochemical markers in the brains of the fostered offspring by immunohistochemical analyses. This was conducted in adult tissue because the functional effects of prenatal immune challenge are expected to achieve their prominence when the affected individuals reach adult age (see eg Zuckerman et al, 2003).

\section{MATERIALS AND METHODS}

\section{Subjects}

Female and male C57BL6/J breeders were obtained from our in-house specific pathogen free colony at the age of 10-14 weeks. The breeding procedure and the verification of pregnancy have been fully described elsewhere (Meyer $e t a l$, 2005). All procedures described in the present study had been previously approved by the Cantonal Veterinarian's Office of Zurich, and are in agreement with the Principles of Laboratory Animal Care (NIH publication No. 86-23, revised 1985).

\section{Prenatal Treatment}

Pregnant dams on GD 9 received either a single injection of $5 \mathrm{mg} / \mathrm{kg}$ PolyI:C (potassium salt; Sigma Aldrich, Switzerland) or vehicle (saline) via the intravenous route at the tail vein under mild physical constraint. The dose of PolyI:C was calculated based on the pure form PolyI:C. The volume of injection was $5 \mathrm{ml} / \mathrm{kg}$.

\section{Postnatal Cross-Fostering}

On the day of birth, offspring born to PolyI:C- and vehicletreated dams were culled to litters of 6-8 animals each (with 3-4 animals per sex) and cross fostered to surrogate mothers for postpartum rearing until weaning. The crossfostering procedure has been fully described before (Meyer et al, 2006c). A total of 16 litters (8 PolyI:C and 8 vehicle) were cross-fostered to 16 rearing mothers, half of which had been subjected to PolyI:C exposure during pregnancy and the other half to vehicle treatment only.

\section{Behavioral Testing}

The offspring were weaned and sexed at postnatal day (PND) 24. Littermates of the same sex were caged separately, thereby separating the animals according to the four treatment conditions that corresponds to a $2 \times 2$ (prenatal treatment $\times$ postnatal rearing) factorial design. All animals were maintained under ad libitum food (Kliba, 3430, Klibamühlen, Kaiseraugst, Switzerland) and water diet, and kept in a temperature and humidity-controlled $\left(21 \pm 1^{\circ} \mathrm{C}, 55 \pm 5 \%\right)$ animal vivarium under a $12: 12 \mathrm{~h}$ reversed light-dark cycle (lights off at 0700).

Experiments 1 and 2 assessed the locomotor response to systemic AMPH and MK-801 treatment, respectively, in preadolescent offspring, that is, when the animals reached about 35 days of age. Both experiments were conducted in separate cohorts of drug-naive animals to avoid repeated drug exposure, and included testing of offspring deriving from multiple independent litters formed by the crossfostering procedure (at least six per treatment group). Experiments 3 and 4 tested the locomotor reaction to systemic AMPH and MK-801 administration in adulthood, that is, when the offspring reached PND 100-110. Again, the 
experiments were conducted in separate cohorts of drug-naive animals, and included testing of offspring deriving from multiple litters. Pre-adolescence was defined as the period between PND 28 and 40, and adulthood was defined as the period of PND 65 onward, according to the gradual attainment of sexual maturity and age-specific behavioral discontinuities from younger to older animals (Spear, 2000). These two developmental stages are largely comparable between mice and rats, and roughly correspond to a period between 11-16 year and $\sim 20$ years onward, respectively, in humans (Spear, 2000; Sisk and Foster, 2004; Rasier et al, 2006).

The number of subjects employed in each of the behavioral tests is listed in Table 1. All behavioral testing was carried out in the dark phase of the light-dark cycle.

The behavioral tests were conducted in four identical square arenas $(40 \times 40 \times 35 \mathrm{~cm}$ high $)$ made of wood and painted white as described before (Meyer et al, 2005). They were located in a testing room under dim-diffused lighting (approximately 35 lux as measured in the center of the arenas). A digital camera was mounted directly above the four arenas. Images were captured at a rate of $5 \mathrm{~Hz}$ and transmitted to a PC running the Ethovision (Noldus, The Netherlands) tracking system.

\section{Drugs}

D-Amphetamine sulfate (AMPH; Sigma-Aldrich, Switzerland) and dizocilpine (MK-801; Merck, Sharp \& Dohme, UK) were dissolved in isotonic $0.9 \% \mathrm{NaCl}$ solution to achieve the desired concentration for injection. Our interest to evaluate the effects of the pre- and postnatal manipulations on the locomotor response to systemic AMPH and MK-801 treatment was based on the fact that acute treatment with indirect dopamine-receptor agonists and noncompetitive NMDA-receptor antagonists can induce schizophrenia-like psychotic symptoms in healthy human subjects and exacerbate psychotic symptoms in schizophrenic patients (eg Lieberman et al, 1987; Krystal et al, 1994; Lahti et al, 1995; Laruelle et al, 1996). AMPH and MK801 were administered via the intraperitoneal (i.p.) route at a dose of 2.5 and $0.15 \mathrm{mg} / \mathrm{kg}$, respectively. The dose of
AMPH was chosen based on our previous results of the drug's effect on open field locomotor activity in animals subjected to prenatal PolyI:C exposure (Meyer et al, 2005). The dose of MK-801 was chosen based on previous dose response studies in C57BL/6 mice (Wu et al, 2005). The volume of injection was $5 \mathrm{ml} / \mathrm{kg}$ for both drugs. All solutions were freshly prepared on the day of testing.

\section{Behavioral Testing Procedures}

To acclimatize the animals to the open field, they were placed in the center of the arena, and allowed to explore freely for $60 \mathrm{~min}$. At the end of this time period, the animals were removed from the apparatus and injected with saline solution. They were then immediately returned to the same arenas and allowed to explore for another $60 \mathrm{~min}$. Subsequently, the animals were briefly removed from the apparatus once more, administered with either AMPH or MK-801, and returned to the same arenas again. The locomotor responses to the acute drug challenges were then monitored for a period of $120 \mathrm{~min}$.

Locomotor activity was indexed by the distance traversed in the entire open field arena as a function of $10 \mathrm{~min}$ bins. The data were subjected to parametric analysis of variance (ANOVA) with between-subjects factors of prenatal treatment, postnatal rearing and sex, and with the withinsubjects factor of time bins. The data collected on the three phases of the experiment (ie no treatment, vehicle treatment, and drug treatment) were analyzed separately.

\section{Immunohistochemistry}

Dopamine-related immunohistochemical analyses in the brains of the fostered offspring included the quantification of immunoreactivities (IR) for tyrosine hydroxylase (TH) and for dopamine D1 and D2 receptors (D1R and D2R, respectively) to assess both pre- and postsynaptic dopaminergic markers. Glutamate-related neurochemical markers were studied by immunohistochemical analyses of the NMDA-receptor subunit 1 (NR1) and the GluR1 subunit of the alpha-amino-3-hydroxyl-5-methyl-4-isoxazole propionic

Table I Summary of the Number of Subjects Employed in the Behavioral and Neuroanatomical Assays

Experimental groups (prenatal treatment-postnatal rearing)

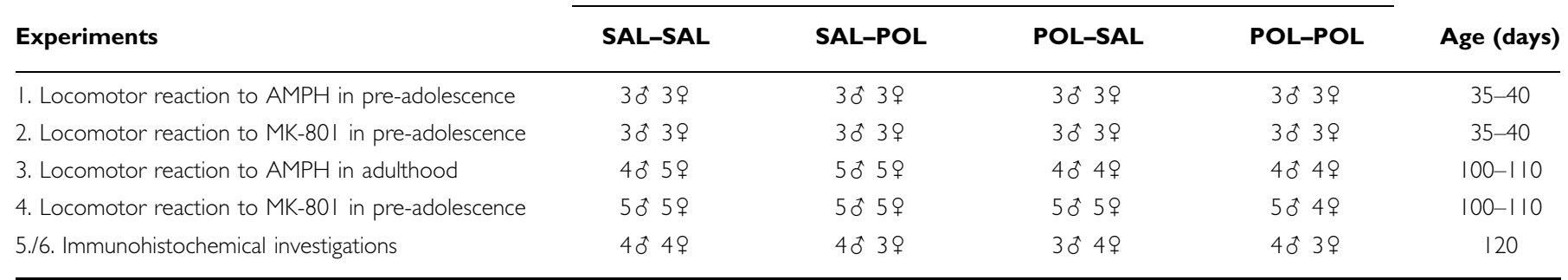

Abbreviations: AMPH, amphetamine; POL, Polyl:C; SAL, saline.

Neonates of saline (SAL)- and Polyl:C (POL)-treated dams were cross fostered to surrogate-rearing mothers, which had been exposed to either saline or Polyl:C during pregnancy. This yielded four experimental groups according to a two (prenatal treatment) $\times$ two (postnatal rearing) design. Experiments I and 2 assessed the locomotor reaction to systemic AMPH and dizocilpine (MK-80I) administration in preadolescence, respectively. Experiments 3 and 4 tested the offspring's reaction to these drugs in adulthood. In addition, dopamine- and glutamate-related immunohistochemical analyses were conducted in the brains of adult offspring (Experiments 5 and 6). All behavioral tests (Experiments I-4) were conducted in separate cohorts of drug-naive animals to avoid repeated drug exposure. The immunohistochemical analyses were also performed in a separate cohort of drug-naive animals. 
acid (AMPA) receptor (GluR1). The concomitant evaluation of both glutamate receptor types is warranted especially if one aims at relating potential differences in the sensitivity to noncompetitive NMDA-receptor antagonist such as MK-801 to putative changes in glutamate receptor expression. This is because enhanced behavioral responding to noncompetitive NMDA-receptor antagonists has been attributed to a reduced glutamate action at NMDA receptors and, at the same time, to increased glutamate action at non-NMDA receptors, including AMPA receptors (Holcomb et al, 2005). All immunohistochemical analyses were performed in a separate cohort of adult drug-naive animals. The prenatal treatment and postnatal cross-fostering procedures were identical as described above. The number of subjects employed in the postmortem analyses is summarized in Table 1.

At the age of approximately 120 days, mice were anesthetized with an overdose of sodium pentobarbital $(5 \mathrm{mg} / \mathrm{kg}$, i.p.) and perfused transcardially as described before (Meyer et al, 2006b). The dissected brains were postfixed in $4 \%$ phosphate-buffered paraformaldehyde solution containing $15 \%$ picric acid and microwaved in citric acid for $90 \mathrm{~s}$ with an intensity of $480 \mathrm{~W}$. The brains were then cryoprotected using $30 \%$ sucrose in phosphate buffered saline (PBS). This is a standard and highly used procedure established by Fritschy et al (1998), which has been proven to produce highly reliable results in the detection of dopamine- and glutamate-related markers by immunohistochemical methods in our previous pilot study. Coronal sections ( $30 \mu \mathrm{m}$ thick) were cut from frozen blocks with a sliding microtome. Twelve series of sections from bregma +2.10 to $-3.80 \mathrm{~mm}$ were collected, rinsed in phosphate-buffered saline (PBS), and stored at $-20^{\circ} \mathrm{C}$ in antifreeze solution until further processing.

For immunohistochemical staining, the slices were rinsed three times for $10 \mathrm{~min}$ in PBS. Blocking was done in PBS, $0.3 \%$ Triton $\mathrm{X}-100,5 \%$ normal serum for $1 \mathrm{~h}$ at room temperature. The following primary antibodies were used: rabbit anti-D1 (Chemicon, CA, USA; diluted 1:250), rabbit anti-D2 (Chemicon, CA, USA; diluted 1:750), sheep antiTH (Biologicals, Rogers, AR, USA; diluted 1:1000), mouseanti NMDA receptor subunit 1 (NR1) (Bio Reagents, Golden, USA; diluted 1:600), and rabbit-anti AMPA receptor subunit GluR1 (Chemicon; diluted $1: 1000$ ).

All antibodies were diluted in PBS containing $0.3 \%$ Triton $\mathrm{X}-100$ and $2 \%$ normal serum, and the sections were incubated free-floating overnight at room temperature. After three washes with PBS (10 min each), the sections were incubated for $1 \mathrm{~h}$ with the biotinylated secondary antibodies diluted 1:500 in PBS containing 2\% normal serum and $0.3 \%$ Triton X-100. Sections were washed again three times for 10 min in PBS and incubated with Vectastain Kit (Vector Laboratories; Burlingame, CA, USA) diluted in PBS for $1 \mathrm{~h}$. After three rinses in Tris- $\mathrm{HCl} 0.1 \mathrm{M} \mathrm{pH} 7.4$, the sections were stained with $1.25 \%$ 3,3-diaminobenzidine and $0.08 \% \mathrm{H}_{2} \mathrm{O}_{2}$ for 6 to $15 \mathrm{~min}$, rinsed again four times in PBS, dehydrated, and cover-slipped with Eukid (Kindler GmbH, Freiburg, Germany).

\section{Optical Densitometry}

Quantification of all neurochemical markers was achieved by means of optical densitometry using ImageJ software
(ImageJ, NIH, MD, USA) as described before (Meyer et al, 2006b). Optical densitometry was chosen because D1R-, D2R-, TH-, NR1- and GluR1-IR were found to be highly enriched at synaptic sites in the areas of interest (ie dorsal striatum $(\mathrm{dCPu})$, nucleus accumbens, and prefrontal cortex). Digital images were acquired at a magnification of $\times 2.5$ (NA 0.075) using a digital camera (Axiocam MRc5, Zeiss, Jena, Germany) mounted on a Zeiss Axioplan microscope. Exposure times were set so that pixel brightness was never saturated and were held constant during acquisition of all images. Pixel brightness was measured in each brain area of interest by placing two to three rectangular boxes of $100 \times 100 \mu \mathrm{m}$ in the respective area on the right hemisphere. In addition, pixel brightness was measured in the corpus callosum serving as background area. The background-corrected optical densities were averaged per brain region and animal. Eight to ten coronal brain sections per animal from bregma +2.10 to $-1.40 \mathrm{~mm}$ were analyzed.

All immunohistochemical preparations were quantified in the medial prefrontal cortex (mPFC, including cingulate and prelimbic cortices), $\mathrm{dCPu}$, as well as nucleus accumbens core (NAc core) and shell (NAc shell). Here, we focused on these brain areas because of their fundamental but distinct roles in the mediation and modulation of locomotor response to psychostimulant drugs (Creese and Iversen, 1975; Pijnenburg et al, 1976; Heidbreder and Feldon, 1998; Tzschentke, 2001). The data were collected under blind conditions and were subjected to parametric ANOVA with between-subjects factors of prenatal treatment, postnatal rearing, and sex.

\section{Statistical Analysis}

All data were analyzed using parametric ANOVA, followed by Fisher's LSD post hoc comparisons or restricted ANOVAS whenever appropriate. Statistical significance was set at $P<0.05$. All statistical analyses were carried out using the statistical software StatView (version 5.0) implemented on a PC running the Windows XP operating system. Preliminary analyses showed that the four treatment groups did not significantly differ in the spontaneous locomotor activity during the initial acclimatization period to the open field. These data were therefore not included in the final presentation of the results.

\section{RESULTS}

\section{Experiment 1: Locomotor Response to Systemic AMPH in Preadolescence}

Saline injection. There was a clear habituation effect with a reduction of locomotor activity over time, and the four groups were highly comparable (Figure 1). ANOVA only revealed a significant main effect of bins $(F(5,80)=7.40$, $P<0.001)$ with no other main effects or interactions attaining statistical significance.

Amphetamine injection. Administration of AMPH resulted in a marked increase in locomotor activity, which peaked at about $40 \mathrm{~min}$ post-injection. This led to the presence of a significant main effect of bins $(\mathrm{F}(11,176)=3.95, P<0.001)$ 
in the ANOVA of distance traveled. Most importantly, the locomotor enhancing effects of AMPH were increased in offspring subjected to prenatal PolyI:C exposure regardless of the postnatal rearing condition and sex (Figure 1a). These impressions were supported by a significant prenatal treatment $\times$ bins interaction term $(\mathrm{F}(11,176)=2.62, P<0.01)$. Subsequent ANOVAs restricted to each of the $10 \mathrm{~min}$ bins were then conducted. These analyses showed that the efficacy of prenatal PolyI: $\mathrm{C}$ exposure to enhance the locomotor reaction to systemic AMPH in pre-adolescent animals was statistically significant in the second $(\mathrm{F}(1,16)=5.12, P<0.05)$ and third $(\mathrm{F}(1,16)=4.47, P<0.05) 10 \mathrm{~min}$ bins after drug injection in comparison with prenatal vehicle treatment (Figure 1b). No other main effects or interactions attained statistical significance.

\section{Experiment 2: Locomotor Response to Systemic MK-801 in Pre-adolescence}

Saline injection. Consistent with the results obtained in Experiment 1, no significant differences were detected between the four experimental groups in the locomotor response to saline treatment (Figure 2). The habituation effect was present in all animals independent of prenatal treatment and postnatal rearing, leading to the presence of a significant main effect of bins $(\mathrm{F}(5,95)=10.05, P<0.001)$ in the ANOVA of distance traversed. No other main effects or interactions attained statistical significance.

MK-801 injection. Juvenile offspring displayed only a weak locomotor reaction to systemic MK-801 treatment, and the peak response to the drug differed between male and female subjects regardless of prenatal treatment and postnatal rearing. The locomotor reaction to $\mathrm{MK}-801$ in males peaked at about $40 \mathrm{~min}$ post-injection and gradually subsided afterward. Conversely, the peak response in females was obtained at about 100 min post-injection (Figure $2 \mathrm{~b}$ ). These patterns of results led to the emergence of a significant sex $\times$ bins interaction term $(F(11,209)=3.60, P<0.001)$ in the ANOVA of distance traveled. However, no main effects or interactions involving the between-subject factors of prenatal treatment or postnatal rearing attained statistical significance. Hence, the four experimental groups did not differ significantly in the locomotor response to systemic MK-801 treatment in preadolescence (Figure 2a).

\section{Experiment 3: Locomotor Response to Systemic AMPH in Adulthood}

Saline injection. Similar to the results obtained in preadolescence, no significant differences were obtained between the four treatment groups in the locomotor reaction to saline administration (Figure 3). Locomotor activity decreased over time, leading to a significant main effect of bins $(\mathrm{F}(5,135)=2.94, P<0.05)$ in the ANOVA of distance traversed. No other main effects or interactions attained statistical significance.

Amphetamine injection. Systemic AMPH administration in adulthood resulted in an overall increase in locomotor activity, which peaked at about $30 \mathrm{~min}$ post-injection and gradually subsided afterward. In comparison to prenatal control animals that were raised by vehicle-treated surrogate mothers (ie saline-saline (SAL-SAL offspring), the locomotor enhancing effects of AMPH were potentiated in prenatal control offspring that were adopted by immunechallenged surrogate mothers (ie in saline-PolyI:C (SALPOL) offspring), as well as in prenatally PolyI:C-exposed adult offspring regardless of the postnatal rearing condition (ie in POL-SAL and POL-POL animals). These effects emerged independently of sex (Figure 3). Statistical support for these impressions were obtained by the presence of a significant main effect of bins $(\mathrm{F}(11,297)=65.34, P<0.001)$, as well as by the significant bins $\times$ prenatal treatment $\times$ postnatal rearing interaction $(\mathrm{F}(11,297)=3.13$, $P<0.01)$ in the ANOVA of distance traveled. No other
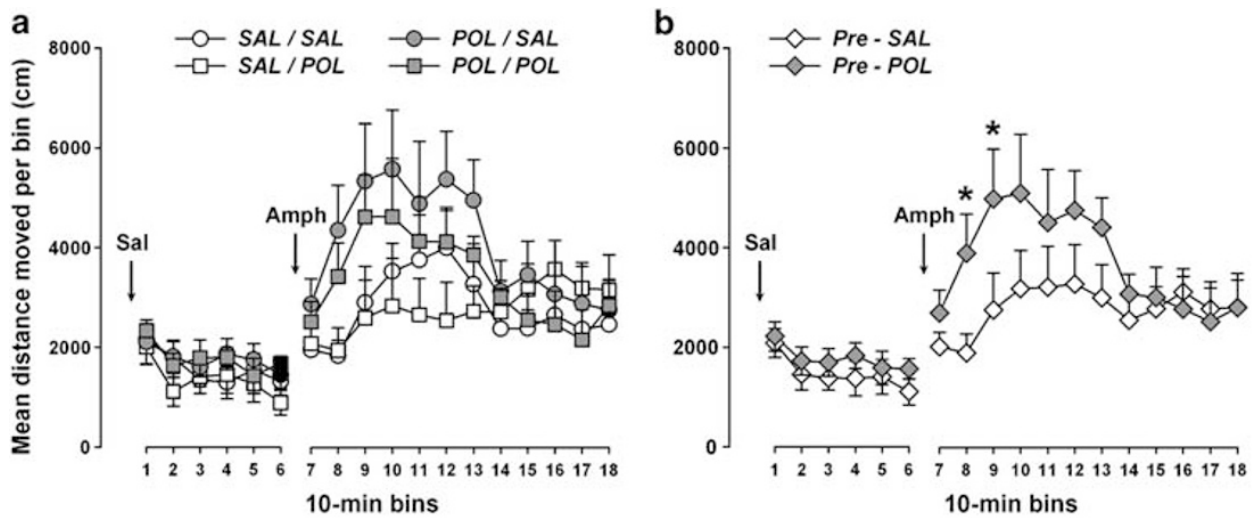

Figure I The effects of the prenatal and postnatal manipulations on the locomotor reaction to systemic AMPH (2.5 mg/kg, i.p.) treatment in preadolescence. (a) Prenatal Polyl:C administration increased the locomotor-stimulating effects of AMPH in pre-adolescent animals as assessed by the distance traversed in the open field. This effect was largely independent of the postnatal rearing condition, that is, it emerged similarly in prenatally Polyl:Cexposed offspring that were raised by either vehicle-exposed ( $\mathrm{POL}-\mathrm{SAL}$ offspring) or immune-challenged surrogate mothers (POL-POL offspring) relative to prenatal control animals that were raised by vehicle-exposed (SAL-SAL offspring) or immune-challenged surrogate mothers (SAL-POL offspring). (b) The additional comparison restricted to prenatal treatment further emphasizes that the enhanced locomotor reaction to systemic AMPH in prenatally Polyl:C exposed offspring (Pre-POL) relative to prenatal control animals (Pre-SAL) was most pronounced between 20 and 40 min post-injection. Asterisk (*) refers to a statistical significance of $P<0.05$ associated with the main effect of prenatal treatment in the randomized block ANOVAs conducted at each of the 10 min bins. The number of subjects in each group is listed in Table I. All values refer to mean + SEM. 

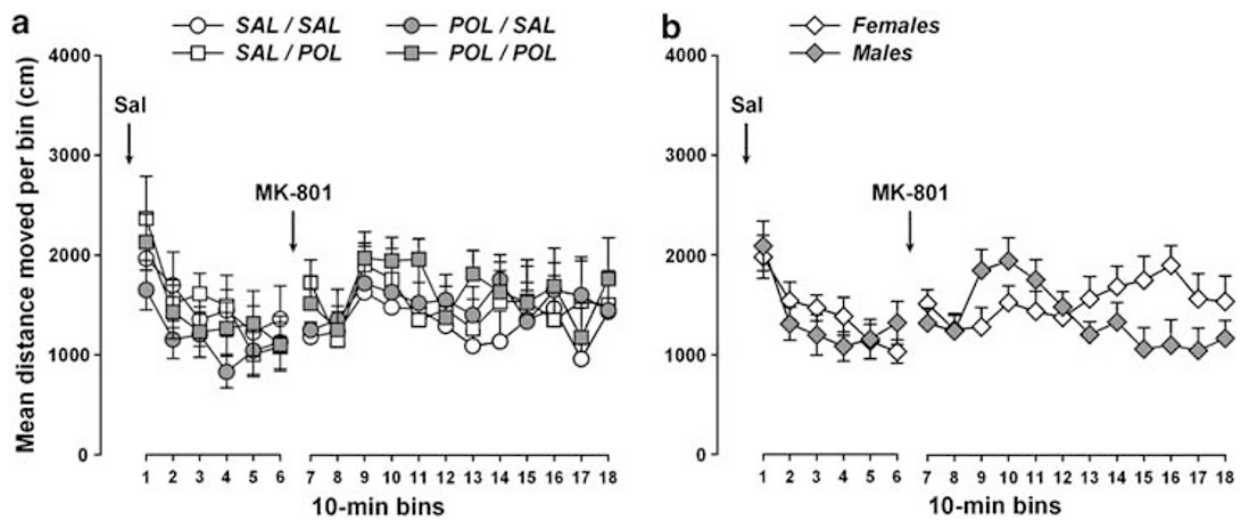

Figure 2 The effects of the pre- and postnatal manipulations on the locomotor reaction to systemic dizocilpine (MK-80 I; $0.15 \mathrm{mg} / \mathrm{kg}$, i.p.) treatment in preadolescence. (a) The locomotor response to acute MK-80I treatment was generally low in pre-adolescent animals. No significant differences were detected between the four experimental groups, that is, between prenatally Polyl:C-exposed animals that were either adopted by vehicle-treated surrogate mothers (POL-SAL offspring) or immune-challenged rearing mothers (POL-POL offspring), and prenatal control animals that were raised by vehicle-treated surrogate mothers (ie SAL-SAL offspring) or Polyl:C-exposed surrogate mothers (SAL-POL offspring). (b) The peak response to the drug differed between male and female subjects regardless of prenatal treatment and postnatal rearing. The number of subjects in each group is listed in Table I. All values refer to mean \pm SEM.

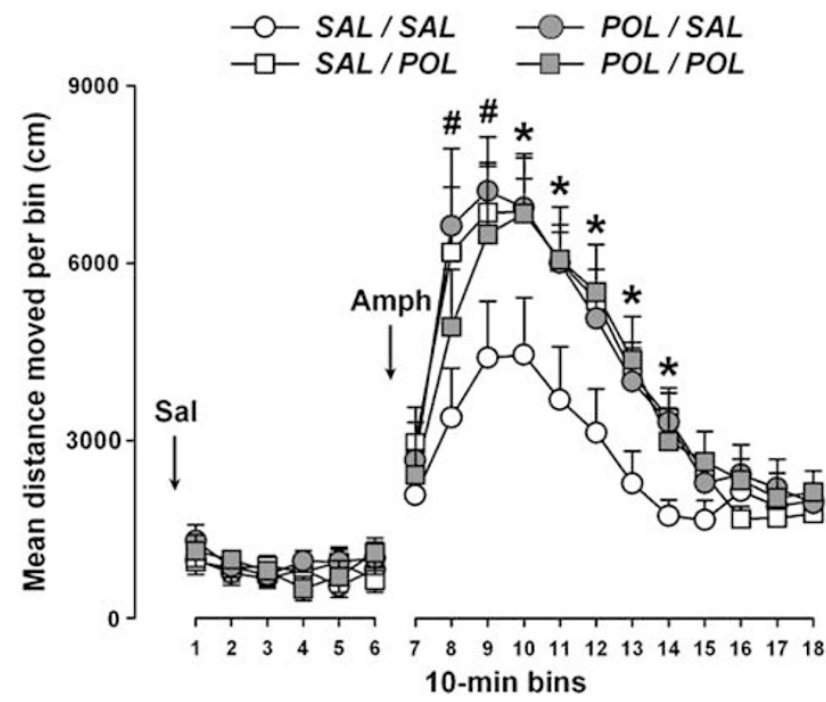

Figure 3 The effects of the prenatal and postnatal manipulations on the locomotor reaction to systemic AMPH $(2.5 \mathrm{mg} / \mathrm{kg}$, i.p.) treatment in adulthood. In comparison to prenatal control animals that were raised by saline (SAL)-treated surrogate mothers (ie SAL-SAL offspring), the locomotor reaction to systemic AMPH was enhanced in prenatal control animals that were adopted by Polyl:C-exposed surrogate mothers (ie in SAL-POL offspring), and in offspring subjected to prenatal Polyl:C exposure independent of the postnatal rearing condition (ie in $\mathrm{POL}-\mathrm{SAL}$ and POL-POL offspring). Symbols (\#) refer to a statistically significant difference between POL-SAL and SAL-SAL offspring $(P<0.05)$, and $(*)$ to a statistically significant increase in $\mathrm{AMPH}$-induced activity displayed by $S A L-P O L, P O L-S A L$, and $P O L-P O L$ offspring relative to $S A L-S A L$ subjects $\left(P_{S}<0.05\right)$, based on Fisher's LSD post hoc comparisons between the four experimental groups at each of the 10 min bins. The number of subjects in each group is listed in Table I. All values refer to mean + SEM.

main effects or interactions reached statistical significance. Subsequent post hoc comparisons between the four groups at each of the $10 \mathrm{~min}$ bins confirmed the statistically significant difference between SAL-SAL and POL-SAL animals in the second and third bins of drug treatment (all $P<0.05$ ), as well as between SAL-SAL and all other groups within bins four to eight after AMPH administration (all $P<0.05$ ).

\section{Experiment 4: Locomotor Response to Systemic MK-801 in Adulthood}

Saline injection. Consistent with the results obtained before, there was a clear habituation effect with a reduction of locomotor activity over time, and the four groups were highly comparable (Figure 4). ANOVA only revealed a significant main effect of bins $(F(5,155)=6.14, P<0.001)$, and no main effects or interactions involving the betweensubject factors of sex, prenatal treatment, and postnatal rearing attained statistical significance.

MK-801 injection. Locomotor activity gradually increased over time following systemic administration of MK-801. In males, the peak response to the drug challenge was detected at about $90 \mathrm{~min}$ post-injection, and the highest levels of locomotor activity in females were obtained only at the end of the test (ie at about 120 min post-injection; Figure 4c). This led to the presence of a significant main effect of bins $(\mathrm{F}(11,341)=8.13, P<0.001)$ and its interaction with sex $(\mathrm{F}(11,341)=1.99, P<0.05)$ in the ANOVA of distance traversed. Most importantly, the locomotor-stimulating effects of systemic MK-801 were enhanced in adult offspring subjected to prenatal PolyI:C exposure (Figure 4a), and this effect was independent of the postnatal rearing condition and sex (Figure $4 \mathrm{~b}$ ). Statistical support for these interpretations was obtained by the presence of a significant main effect of prenatal treatment $(\mathrm{F}(1,31)=4.32, P<0.05)$. No other main effects or interactions involving the betweensubject factors of sex, prenatal treatment, and postnatal rearing attained statistical significance.

\section{Experiment 5: Expression of Dopamine-Related Neurochemical Markers in the Adult Brain}

Dorsal striatum. Prenatal PolyI:C exposure increased THIR in the $\mathrm{dCPu}$ regardless of the postnatal rearing condition. 

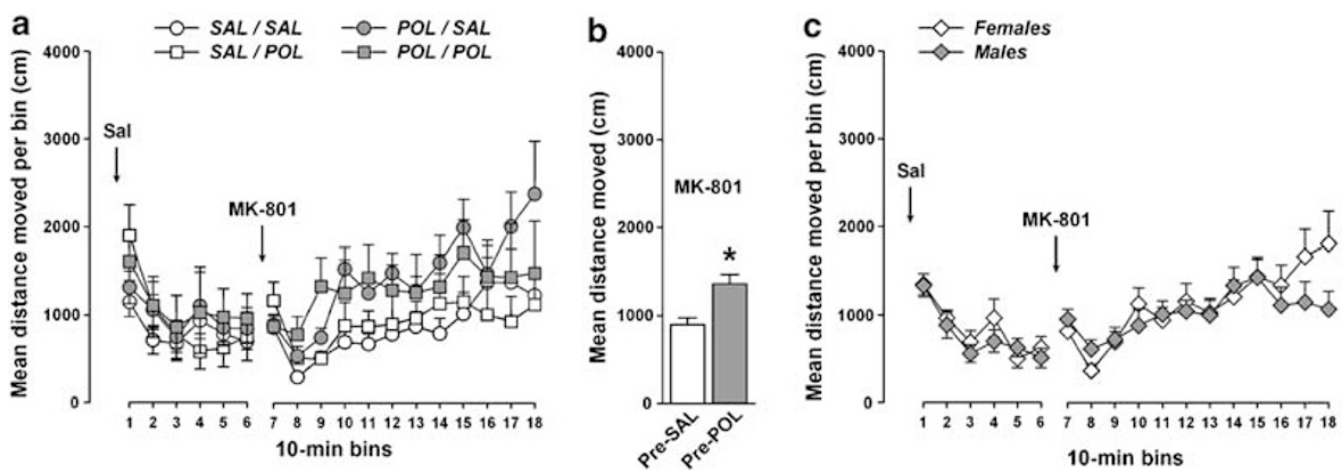

Figure 4 The effects of the prenatal and postnatal manipulations on the locomotor reaction to systemic dizocilpine (MK-80 I; $0.15 \mathrm{mg} / \mathrm{kg}$; i.p.) treatment in adulthood. (a) Prenatal Polyl:C exposure increased the locomotor response to MK-80I in adulthood compared to prenatal vehicle (saline) treatment. This effect was independent of the postnatal rearing condition, that is, it emerged both in prenatally Polyl:C-treated animals that were either adopted by vehicletreated surrogate mothers (POL-SAL) or immune-challenged surrogate mothers (POL-POL). Prenatal control animals that were raised by saline-treated surrogate mothers (SAL-SAL) or by Polyl:C-exposed surrogate mothers (SAL-POL) showed equally low reactions to the drug treatment. (b) The bar diagram highlights the effect of prenatal Polyl:C exposure (Pre-POL) on enhancing the locomotor response to MK-80I relative to prenatal saline treatment (Pre-SAL). (c) The overall peak response to the drug was obtained earlier in males than females regardless of prenatal treatment and postnatal rearing. Symbol (*) refers to a statistical significance of $P<0.05$ associated with the main effect of prenatal treatment revealed by ANOVA. The number of subjects in each group is listed in Table I. All values refer to mean \pm SEM.

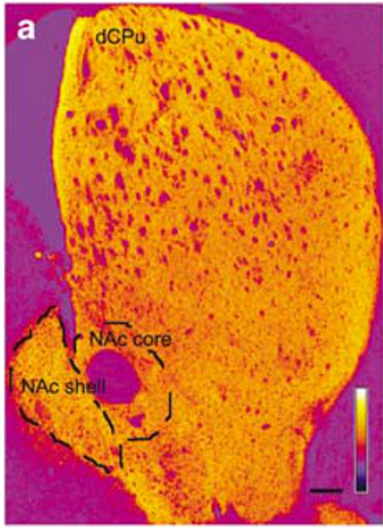

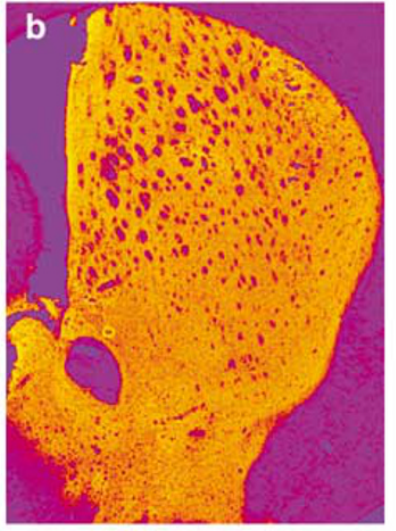

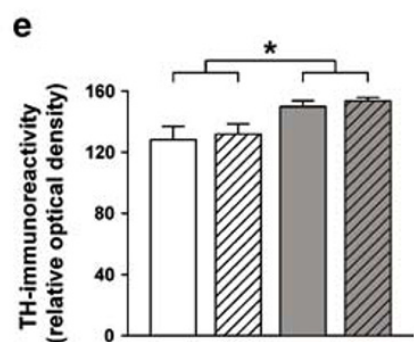

Prenatal: SAL SAL POL POL Postnatal: SAL POL SAL POL
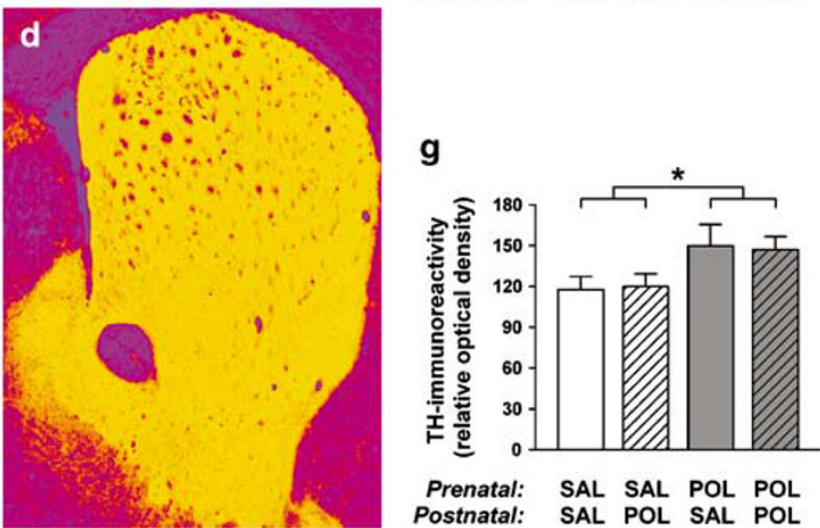
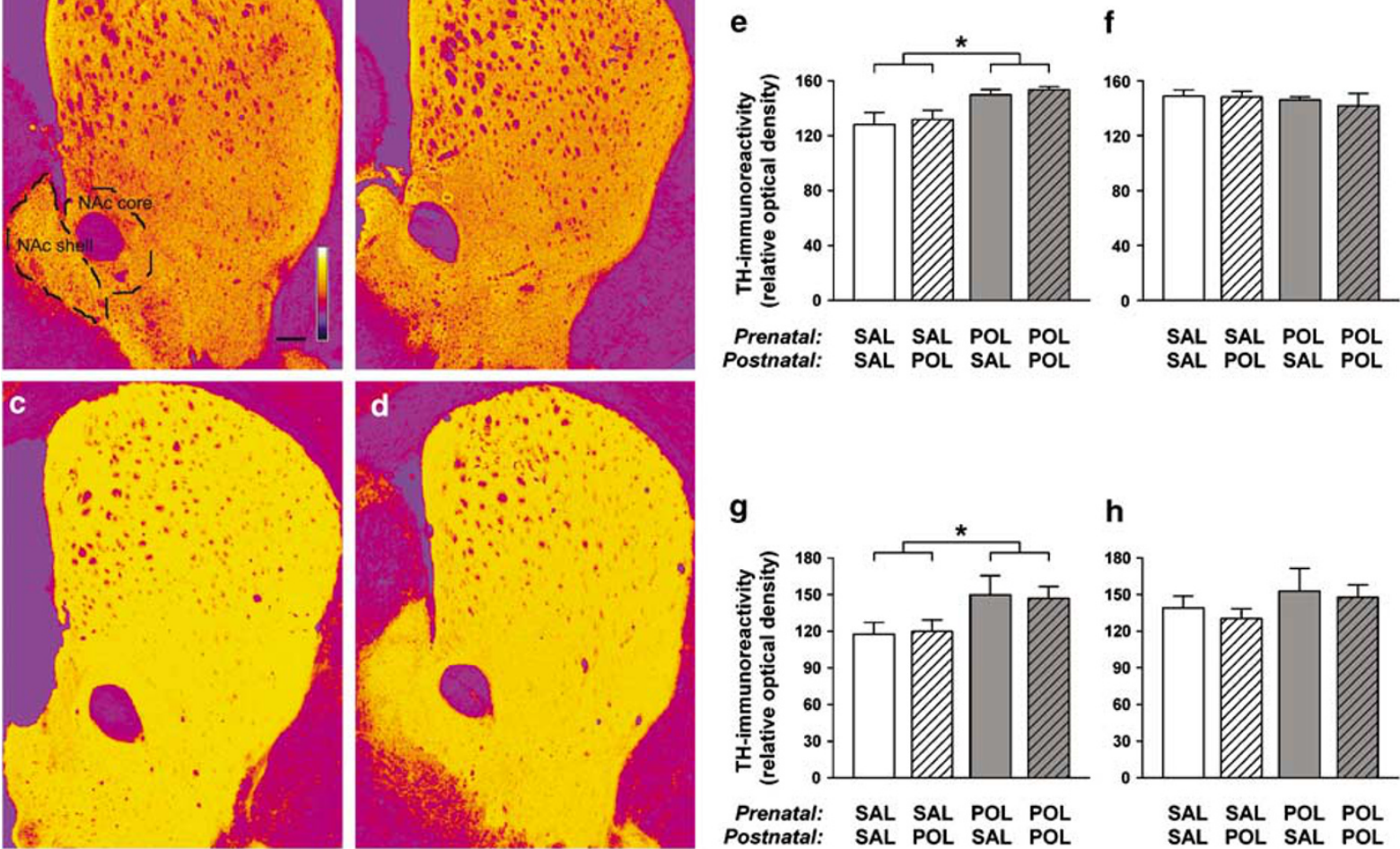

Figure 5 The effects of the prenatal and postnatal manipulations on the expression of TH-IR in striatal structures. Compared to prenatal control animals raised by vehicle-treated surrogate mothers (a) and prenatal control offspring adopted by immune-challenged rearing mothers (b), the prenatal exposure to Polyl:C enhanced striatal TH-IR in adult offspring raised by vehicle-treated (c) and immune-challenged surrogate mothers (d). This effect was significant in the $\mathrm{dCPu}$ of male animals subjected to prenatal Polyl:C exposure (e) but not in female subjects (f). Prenatal Polyl:C exposure also increased TH-IR in the NAc shell $(\mathrm{g})$ relative to prenatal vehicle treatment, and this effect was independent of the postnatal rearing condition and sex. TH-IR in the NAc core ( $h$ ) was not significantly affected by the prenatal and postnatal manipulations in both female and male subjects. Strongest staining intensities in (a) are shown in white/ yellow, while the background is represented in dark purple (bar inset in a). Scale bar in (a) $=200 \mu \mathrm{m}$. Symbol (*) in (e) and (g) refers to a statistical significance of $P<0.05$ associated with the main effect of prenatal treatment revealed by ANOVA. The number of subjects in each group is listed in Table I. All values refer to mean \pm SEM. 
However, this effect only attained statistical significance in male offspring exposed to prenatal immune challenge, but not in female subjects (Figure 5). This led to the presence of a significant prenatal treatment $\times$ sex interaction $(\mathrm{F}(1,21)=4.79, P<0.05)$ in the ANOVA of TH-IR in the dCPu. Additional ANOVAs restricted to either sex confirmed that prenatal PolyI:C exposure increased TH-IR in the $\mathrm{dCPu}$ of male offspring independent of postnatal rearing (main effect of prenatal treatment: $\mathrm{F}(1,10)=4.31, P<0.05$ ), but not in females $(\mathrm{F}<1, \mathrm{NS})$.

The immunoreactivities for $\mathrm{D} 1 \mathrm{R}$ and $\mathrm{D} 2 \mathrm{R}$ in the $\mathrm{dCPu}$ were not significantly affected by either manipulation. Hence, D1R- and D2R-IRs in the $\mathrm{dCPu}$ were highly comparable among the four treatment groups. The means \pm SEM of D1R- and D2R-IRs for each experimental group are provided in Table 2 .

Shell subregion of nucleus accumbens. Prenatal immune activation also led to an elevation of TH-IR in NAc shell regardless of the postnatal adoption procedure. Here, this effect was independent of sex (Figure 5). ANOVA of TH-IR in NAc shell yielded a significant main effect of prenatal treatment $(\mathrm{F}(1,21)=4.54, P<0.05)$, and no other main effects or interaction terms attained statistical significance. Again, no significant differences in D1R- and D2R-IRs in NAc shell were detected between the four groups (see Table 2).

Core subregion of nucleus accumbens. In contrast to the effects on TH-IR in NAc shell, prenatal PolyI:C exposure did not significantly alter TH-IR in NAc core (Figure 5).
Similarly, no main effects or interactions involving postnatal rearing and sex were revealed in the statistical analyses of TH-IR in NAc core. Hence, neither the prenatal nor postnatal manipulations significantly affected TH-IR in the NAc core. The immunoreactivities for D1R and D2R in NAc core were also not significantly affected by either pre- or postnatal manipulation (see Table 2).

Medial prefrontal cortex. TH-IR in the MPFC was generally low and largely indistinguishable from background staining. It was therefore not subjected to quantification and statistical analyses.

Prenatal immune activation significantly decreased D1R-IR in the mPFC irrespective of the postnatal rearing condition. However, this effect only emerged in male but not female subjects (Figure 6), leading to a significant interaction between prenatal treatment and sex in the ANOVA of this measure $(F(1,21)=5.52, P<0.05)$. Subsequent ANOVAs restricted to either sex verified the significant PolyI:C-induced reduction of D1R-in the mPFC of male (main effect of prenatal treatment: $\mathrm{F}(1,10)=5.30$, $P<0.05)$ but not of female offspring $(\mathrm{F}<1, \mathrm{NS})$.

Prenatal PolyI:C exposure also significantly reduced D2RIR in the MPFC of male but not female offspring, and this effect emerged independently of the postnatal adoption procedure (Figure 7). This led to a significant interaction between prenatal treatment and sex in the ANOVA of D2RIR in the $\operatorname{mPFC}(\mathrm{F}(1,21)=8.30, P<0.01)$. Subsequent ANOVAs restricted to either sex confirmed that prenatal immune activation reduced $\mathrm{mPFC}$ D2R-IR in male (main

Table 2 Summary of the Effects of the Pre- and Postnatal Manipulations on the Corticostriatal Immunoreactivity of Dopamine-Related Neurochemical Markers in Adulthood

Experimental groups (prenatal treatment-postnatal rearing)

\begin{tabular}{|c|c|c|c|c|}
\hline \multirow[b]{2}{*}{ Marker } & \\
\hline & SAL-SAL & SAL-POL & POL-SAL & POL-POL \\
\hline \multicolumn{5}{|l|}{ Tyrosine hydroxylase } \\
\hline Dorsal striatum $^{\mathrm{a}}$ & $138.5 \pm 6.6$ & $|40| \pm 5.3$. & $147.9 \pm 3.0$ & $147.5 \pm 5.5$ \\
\hline Nucleus accumbens core & $139.0 \pm 9.7$ & $130.2 \pm 8.8$ & $152.6 \pm 18.7$ & $147.7 \pm 10.1$ \\
\hline Nucleus accumbens shell & $117.6 \pm 9.6$ & $119.9 \pm 9.3$ & $149.8 \pm 15.7$ & $146.8 \pm 9.7$ \\
\hline Medial prefrontal cortex & $\mathrm{nq}$ & $\mathrm{nq}$ & $\mathrm{nq}$ & $\mathrm{nq}$ \\
\hline \multicolumn{5}{|l|}{ Dopamine $d /$ receptor } \\
\hline Dorsal striatum & $147.9 \pm 1.8$ & $\mid 48.1 \pm 2.3$ & $146.4 \pm 2.8$ & $148.5 \pm 1.7$ \\
\hline Nucleus accumbens core & $154.8 \pm 4.4$ & $146.5 \pm 9.8$ & $153.4 \pm 3.5$ & $154.7 \pm 3.3$ \\
\hline Nucleus accumbens shell & $105.6 \pm 8.2$ & $101.9 \pm 9.1$ & $103.0 \pm 7.4$ & $100.2 \pm 4.1$ \\
\hline Medial prefrontal cortex ${ }^{\mathrm{b}}$ & $36.9 \pm 2.2$ & $37.1 \pm 2.1$ & $32.5 \pm 4.4$ & $33.4 \pm 2.3$ \\
\hline Nucleus accumbens shell & $10.2 \pm 2.2$ & $13.3 \pm 3.5$ & $12.5 \pm 3.0$ & $11.5 \pm 3.1$ \\
\hline Medial prefrontal cortex ${ }^{c}$ & $26.8 \pm 1.3$ & $28.9 \pm 2.1$ & $24.9 \pm 2.0$ & $24.2 \pm 1.6$ \\
\hline
\end{tabular}

Abbreviations: nq, not quantifiable; POL, Polyl:C; SAL, saline.

The number of subjects in each group is listed in Table I. All values refer to mean \pm SEM of relative optical density.

a,b,c Please refer to Figures 5-7 for sex-dependent effects. 

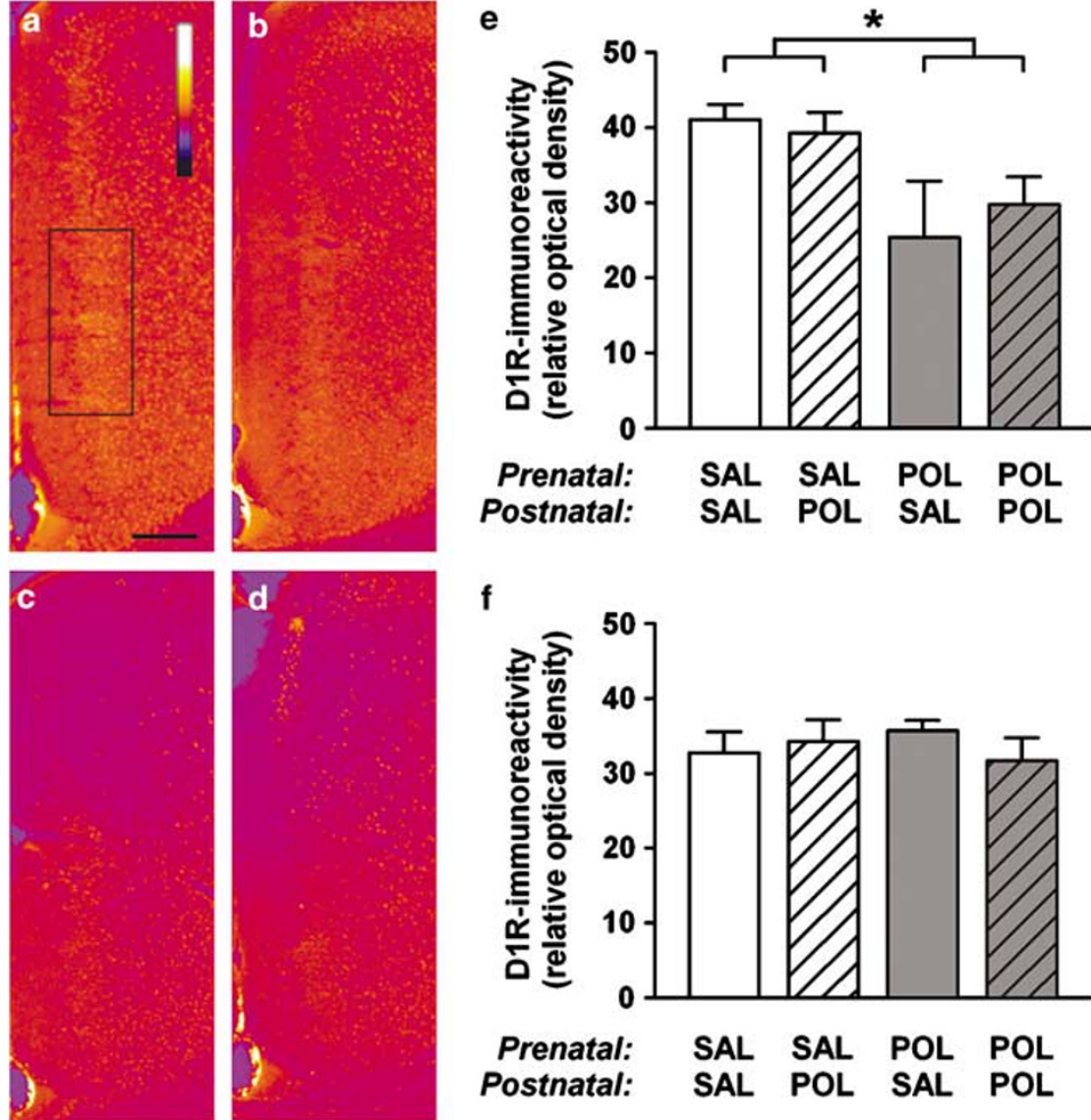

\section{Prenatal: Postnatal: \\ SAL POL SAL POL}

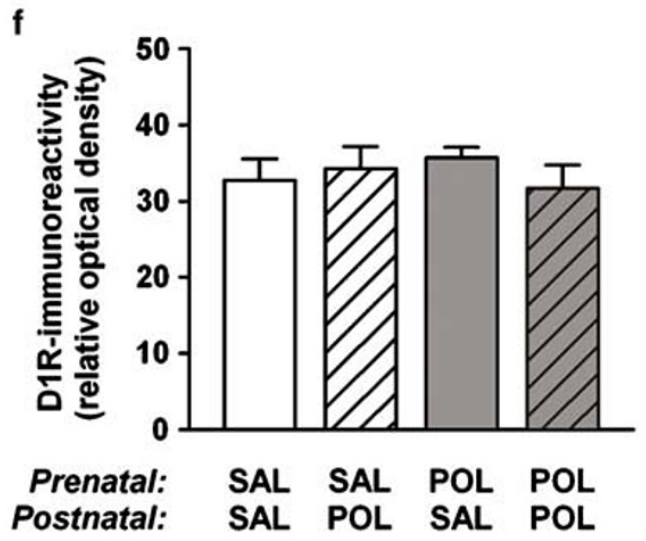

Figure 6 The effects of the prenatal and postnatal manipulations on the expression of DIR-IR in the mPFC. Compared to prenatal control animals raised by vehicle-treated surrogate mothers (a) and prenatal control offspring adopted by immune-challenged rearing mothers (b), prenatal Polyl:C administration decreased DIR-IR in the mPFC (indicated by the square in (a)) of adult offspring raised by vehicle-treated (c) and immune-challenged surrogate mothers (d). However, this effect only emerged in male (e) but not female offspring ( $f$ ). Strongest staining intensities in (a) are shown in white/yellow, while the background is represented in dark purple (bar inset in (a)). Scale bar in (a) $=200 \mu \mathrm{m}$. Symbol (*) in (e) refers to a statistical significance of $P<0.05$ associated with the main effect of prenatal treatment revealed by ANOVA restricted to male subjects. The number of subjects in each group is listed in Table I. All values refer to mean \pm SEM.

effect of prenatal treatment: $\mathrm{F}(1,10)=8.53, P<0.05)$ but not in female offspring $(\mathrm{F}<1, \mathrm{NS})$.

\section{Experiment 6: Expression of Glutamate-Related Neurochemical Markers in the Adult Brain}

Dorsal striatum. Prenatal control animals that were raised by PolyI:C-treated surrogate mothers (ie SAL-POL animals) displayed an increase in GluR1-IR in the dCPu relative to all other groups (Figure 8). This effect was independent of sex. ANOVA of GluRl-IR in the dCPu revealed a significant interaction between prenatal treatment and postnatal rearing $(\mathrm{F}(1,21)=7.96, \quad P<0.05)$. Subsequent post hoc analyses between the four groups confirmed the statistically significant differences between SAL-POL animals and all other groups (all $P<0.05$ ). On the other hand, NR1-IR in the dCPu was not significantly affected by any treatment (see Table 3 ).

Shell subregion of nucleus accumbens. Prenatal PolyI:C exposure reduced GluR1-IR in the NAc shell of female but not male subjects, and this effect emerged independently of the postnatal rearing condition (Figure 9). ANOVA of this measure yielded a significant interaction between sex and prenatal treatment $(\mathrm{F}(1,21)=6.1, P<0.05)$. Subsequent ANOVAs restricted to either sex further verified that prenatal PolyI:C exposure reduced NAc shell GluR1-IR in female (main effect of prenatal treatment: $\mathrm{F}(1,21)=7.96$, $P<0.05)$ but not in male offspring $(\mathrm{F}<1, \mathrm{NS})$. Neither the prenatal nor postnatal manipulation significantly affected the expression of NR1-IR in NAc shell (Table 3).

Core subregion of nucleus accumbens. Neither GluR1- nor NR1-IR was significantly affected by any treatment in NAc core. The means \pm SEM of both measures are summarized for each experimental group in Table 3.

Medial prefrontal cortex. Similar to NAc core, GluR1- and NR1-IRs in the mPFC were not significantly affected by any manipulation. Both measures were highly comparable among the four groups (see Table 3).

\section{DISCUSSION}

The present study demonstrates that dopamine- and glutamate-related pharmacological abnormalities emerging 

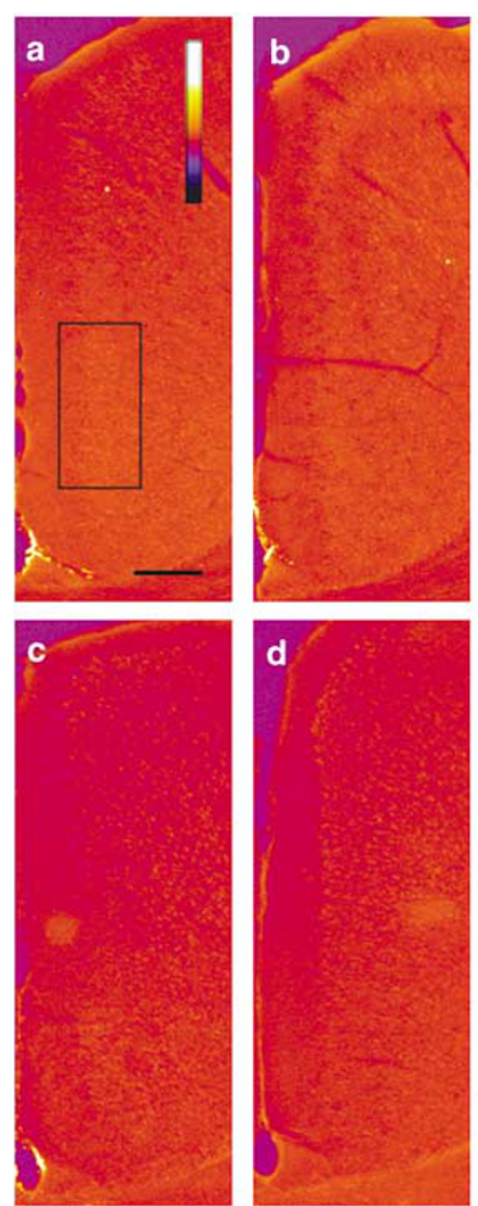

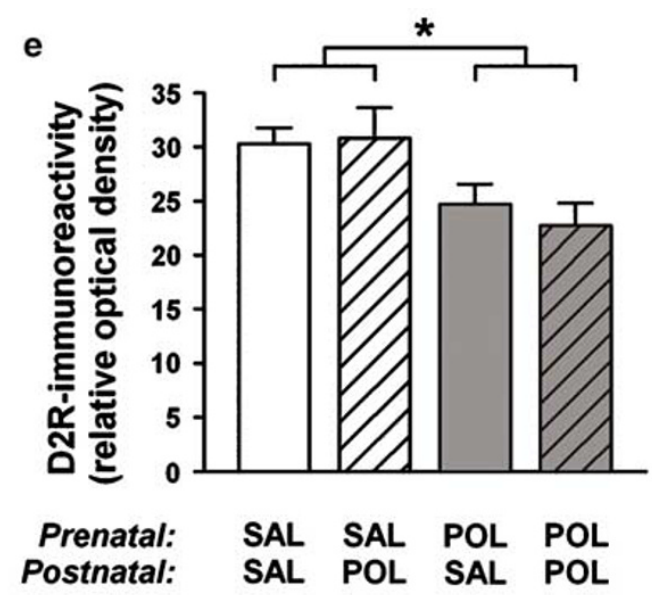

f

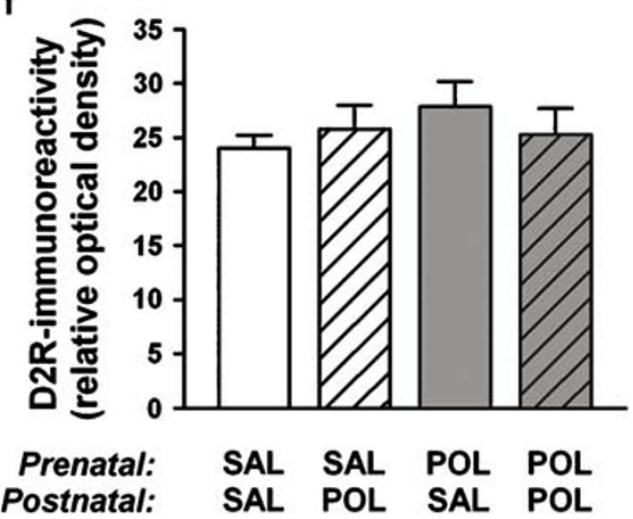

Figure 7 The effects of the prenatal and postnatal manipulations on the expression of D2R-IR in the mPFC. In comparison to prenatal control animals that were raised by vehicle-treated surrogate mothers (a) and prenatal control offspring adopted by immune-challenged rearing mothers (b), prenatal Polyl:C exposure decreased D2R-IR in the mPFC (indicated by the square in (a)) of adult offspring raised by vehicle-treated (c) and immune-challenged surrogate mothers (d). However, this effect was only apparent in male (e) but not female offspring ( $f$ ). Strongest staining intensities in (a) are shown in white/yellow, while the background is represented in dark purple (bar inset in (a)). Scale bar in (a) $=200 \mu \mathrm{m}$. Symbol (*) in (e) refers to a statistical significance of $P<0.05$ associated with the main effect of prenatal treatment revealed by ANOVA restricted to male subjects. All values refer to mean \pm SEM. mPFC, medial prefrontal cortex.

after prenatal immune activation in early/midgestation in mice are readily attributable to prenatal but not postnatal maternal effects on the offspring. We found that prenatal PolyI:C-exposure in early/mid gestation in mice enhanced the sensitivity to the locomotor-stimulating effects of systemic AMPH (Figure 3) and MK-801 treatment (Figure 4) independently of the postnatal adoption procedure, that is, they were equally observed in prenatally immune-challenged animals that were raised by PolyI:C- or vehicletreated surrogate mothers. Hence, the gestational treatment history of the rearing mothers is not a critical determinant of the offspring's potentiated locomotor response to AMPH and MK-801 after prenatal immune activation.

The peak locomotor response to systemic MK-801 differed between juvenile male and female mice (at $\sim 35$ days of age), with peak responses appearing earlier in males than in females (Figure 2). A similar pattern of sex difference was also noticed when adult animals (at $\sim 100$ days of age) were subjected to systemic MK-801 treatment (Figure 4). To the best of our knowledge, the mechanisms underlying the sex-dependent effects on peak responses to systemic MK-801 treatment in mice are unknown to date. Most importantly, however, the prenatal PolyI:C-induced potentiation of MK-801 in adulthood emerged independently of sex. It is thus unlikely that general differences in the peak responses to systemic MK-801 treatment between female and male mice might have undermined our relevant findings, namely that maternal immune activation potentiated MK-801 sensitivity in the adult offspring regardless of the postnatal adoption procedure and sex.

The emergence of enhanced locomotor response to acute AMPH treatment after prenatal immune activation was independent of postnatal age. This contrasts with previous studies in both rats and mice showing that the maternal exposure to PolyI:C during pregnancy potentiated AMPH sensitivity in the resulting offspring only at an adult but not pre-adolescent stage of development (Zuckerman et al, 2003; Ozawa et al, 2006). In the studies by Zuckerman et al (2003) and Ozawa et al (2006), however, PolyI:C was administered to pregnant dams at mid/late gestation (GD 15) and on 6 consecutive days from mid to late gestation (GD 12 -17), respectively. Here, pregnant mice were exposed to a discrete inflammatory reaction in early/mid gestation. Differences in the precise timing of prenatal immune activation may, therefore, offer a possible explanation for the discrepant findings reported here and before (Zuckerman et al, 2003; 

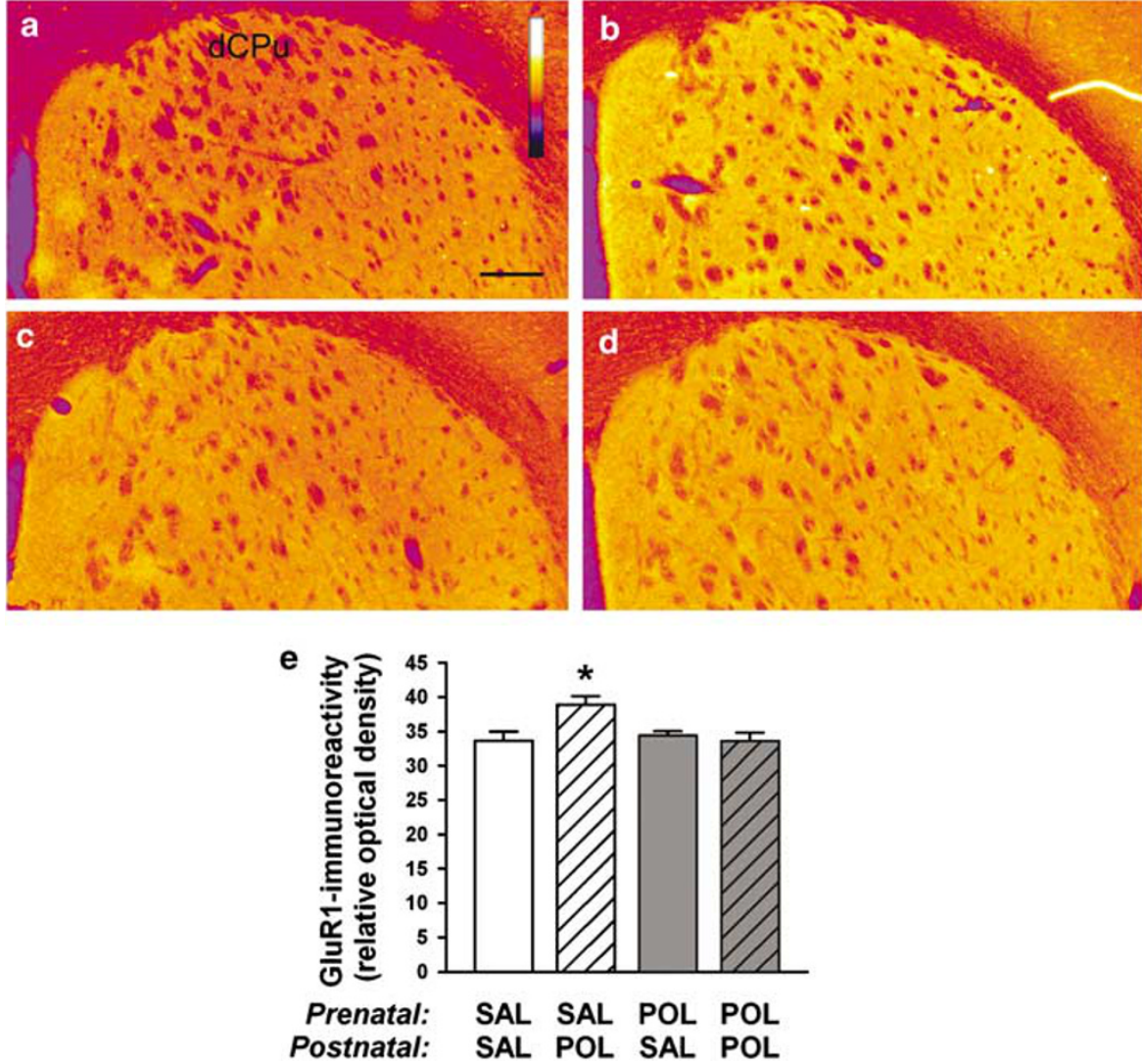

Figure 8 The effects of the prenatal and postnatal manipulations on the expression of GluRI-IR in the dCPu. Prenatal control animals that were raised by Polyl:C-treated surrogate mothers (b) displayed an increase in GluRI-IR in the dCPu (indicated by the dashed line in (a)) relative to prenatal control animals that were raised by vehicle-treated surrogate mothers (a) and prenatally immune-challenged offspring raised by either vehicle-treated (c) or Polyl:C-treated (d) surrogate mothers. This effect emerged independently of sex. Symbol (*) in (e) refers to a statistically significant difference between prenatal control animals that were raised by Polyl:C-treated surrogate mothers and all other groups based on Fisher's LSD post hoc comparison between the four groups. Strongest staining intensities in (a) are shown in white/yellow, while the background is represented in dark purple (bar inset in a). Scale bar in (a) $=200 \mu \mathrm{m}$. The number of subjects in each group is listed in Table I. All values refer to mean \pm SEM. dCPu, dorsal striatum.

Table 3 Summary of the Effects of the Pre- and Postnatal Manipulations on the Corticostriatal Immunoreactivity of Glutamate-Related Neurochemical Markers in Adulthood

Experimental groups (prenatal treatment-postnatal rearing)

\begin{tabular}{|c|c|c|c|c|}
\hline Marker & SAL-SAL & SAL-POL & POL-SAL & POL-POL \\
\hline Dorsal striatum & $39.9 \pm 2.0$ & $42.1 \pm 1.8$ & $42.2 \pm 1.6$ & $40.1 \pm 1.6$ \\
\hline Nucleus accumbens shell & $45.0 \pm 2.1$ & $42.7 \pm 2.0$ & $47.5 \pm 1.8$ & $44.8 \pm 1.2$ \\
\hline Medial prefrontal cortex & $45.1 \pm 1.6$ & $47.1 \pm 2.1$ & $46.5 \pm 2.7$ & $44.5 \pm 1.7$ \\
\hline Dorsal striatum & $33.6 \pm 1.4$ & $39.2 \pm 1.3$ & $34.4 \pm 0.6$ & $33.5 \pm 1.2$ \\
\hline Nucleus accumbens core & $42.7 \pm 1.6$ & $45.4 \pm 1.8$ & $44.4 \pm 1.4$ & $38.7 \pm 1.6$ \\
\hline Nucleus accumbens shell ${ }^{\mathrm{a}}$ & $39.4 \pm 1.7$ & $40.1 \pm 1.9$ & $37.6 \pm 2.2$ & $36.3 \pm 1.8$ \\
\hline Medial prefrontal cortex & $34.9 \pm 3.1$ & $36.1 \pm 2.4$ & $34.8 \pm 2.1$ & $31.4 \pm 2.2$ \\
\hline
\end{tabular}

Abbreviations: NMDA, N-methyl-D-aspartate; POL, Polyl:C; SAL, saline.

The number of subjects in each group is listed in Table I. All values refer to mean \pm SEM of relative optical density.

aPlease refer to Figure 9 for sex-dependent effects. 

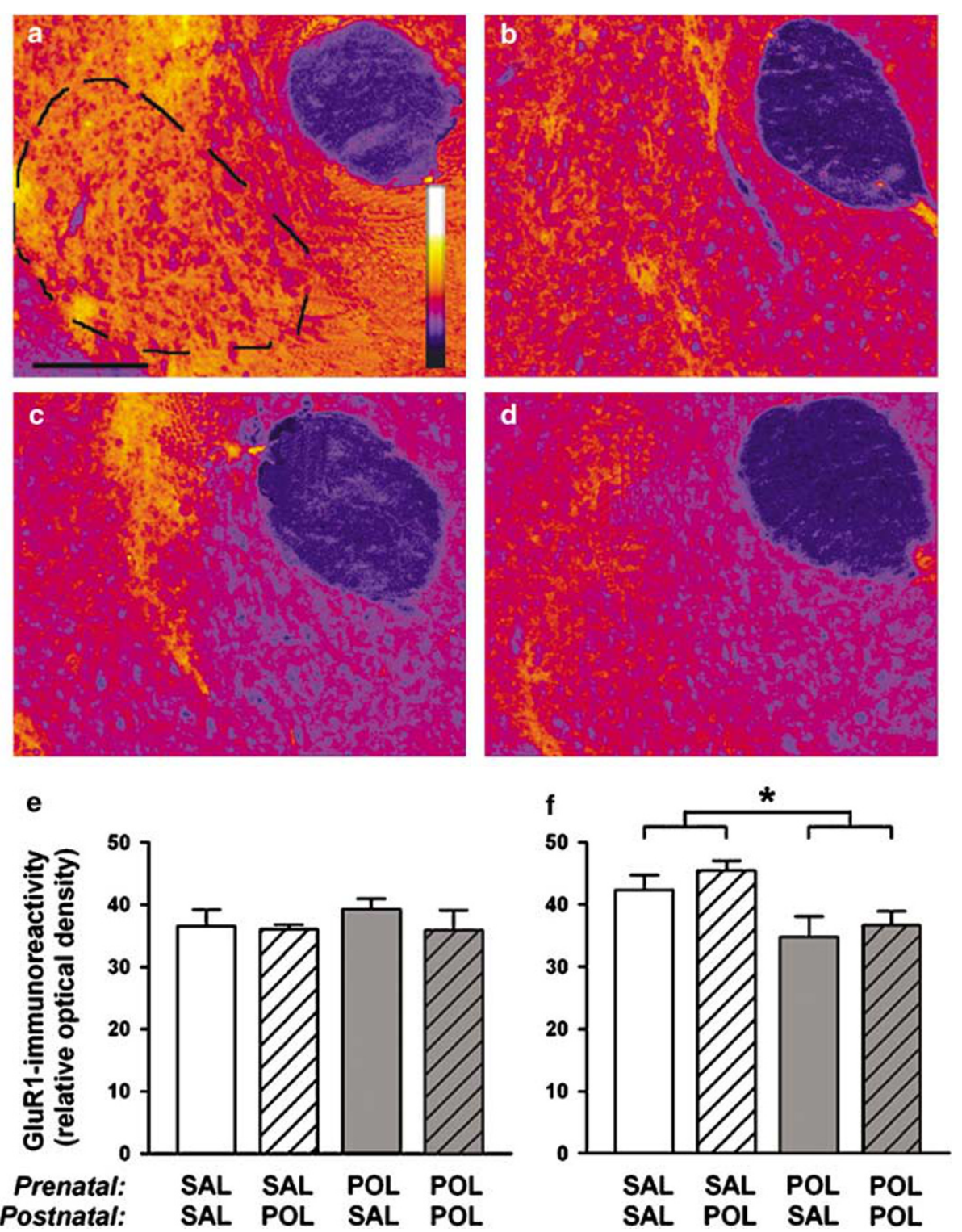

Figure 9 The effects of the pre- and postnatal manipulations on the expression of GluRI-IR in NAc shell. Compared to adult control animals raised by vehicle-treated surrogate mothers (a) and adult control offspring adopted by immune-challenged rearing mothers (b), prenatal Polyl:C administration decreased GluRI-IR in NAc shell (indicated by the dashed line in (a)) of adult offspring raised by vehicle-treated (c) and immune-challenged surrogate mothers (d). However, this effect only emerged in female (f) but not male offspring (e). Strongest staining intensities in (a) are shown in white/yellow, while the background is represented in dark purple (bar inset in (a)). Scale bar in (a) $=200 \mu \mathrm{m}$. Symbol (*) in (e) refers to a statistical significance of $P<0.05$ associated with the main effect of prenatal treatment revealed by ANOVA restricted to female subjects. The number of subjects in each group is listed in Table I. All values refer to mean \pm SEM.

Ozawa et al, 2006). Indeed, by directly comparing the longterm effects between prenatal PolyI:C-induced immune challenge on early/mid (GD 9) and late (GD 17) gestation, we have recently demonstrated significant dissociations between these two prenatal manipulations on the emergence of psychopathological behavior in adulthood (Meyer et al, $2006 \mathrm{a}, \mathrm{b}$ ), as well as on the expression of various neuroanatomical markers in the brains of pre-adolescent offspring (Meyer et al, 2006b). It is thus conceivable that the precise times of prenatal immune activation may also differentially affect the development and/or maturation of the neural substrates involved in locomotor response to systemic AMPH treatment in pre-adolescence.
On the other hand, our findings that maternal PolyI:C exposure on GD9 increased the offspring's locomotor reaction to systemic MK-801 treatment only in adulthood (Figure 4), but not pre-adolescence (Figure 2), readily suggests that post-pubertal maturational processes are involved in the precipitation of this effect. This adds to our previous findings that the disruptive effects of GD9PolyI:C exposure on selective associative learning achieve their prominence when the affected individuals reach adult age (Meyer et al, 2006c). Hence, distinct forms of schizophrenia-related psychopathology are manifested at different stages of postnatal development after the prenatal exposure to inflammatory treatment in early/mid gestation 
in mice: While selective learning deficits (Meyer et al, 2006c) and enhanced responding to acute NMDA-receptor antagonism by MK-801 (Figure 4) only appear at adult age, early fetal brain inflammation leads to enhanced AMPH sensitivity already in pre-adolescence (Figures 1 and 3).

One implication emerging from this contrast is that prenatal immune activation in early/mid pregnancy may induce dopamine-associated endogenous sensitization mechanisms, which precede the onset of the full spectrum of behavioral and pharmacological abnormalities in adulthood. This idea is indeed central for the endogenous sensitization theory of schizophrenia, which suggests that dopaminergic transmission in psychosis-prone individuals may function in a sensitized state already before the onset of psychotic behavior (Laruelle, 2000). One experimental procedure known to induce behavioral sensitization to dopamine-stimulating drugs is withdrawal from repeated AMPH administration (Wolf, 1998; Russig et al, 2005; PelegRaibstein et al, 2006). This manipulation is also known to induce a number of neurochemical adaptations in the central nervous system, including reduced GluR1 expression in the NAc shell (Lu et al, 1997; Lu and Wolf, 1999) and impaired D1R-mediated signaling in the mPFC (Peterson et al, 2006). Our findings of prenatal PolyI:C-induced reduction of GluR1- and D1R-IR in the NAc shell and mPFC, respectively, may thus additionally support the existence of dopamine-associated sensitization mechanism after in utero immune challenge in early/mid gestation. Furthermore, since these neuroanatomical changes emerged regardless of whether prenatally PolyI:C-exposed neonates were reared by immune-challenged or nonchallenged surrogate mothers, our data highlight the possibility that early fetal brain inflammation might represent a relevant etiological factor for postnatal dopamine-associated behavioral sensitization mechanisms.

However, the prenatal PolyI:C-induced down-regulation in NAc shell GluR1-IR and mPFC D1R-IR expression was clearly dependent on the sex of the affected individuals, with the former emerging in female (Figure 9) and the latter in male offspring only (Figure 6). Hence, even though the behavioral reaction to systemic AMPH treatment was highly comparable between male and female animals born to PolyI:C-treated mothers, the neuronal mechanisms underlying the pharmacological effects of prenatal immune activation may critically differ between male and female offspring. In particular, the association between decreased GluR1 in NAc shell and the expression of behavioral sensitization to systemic AMPH (Lu et al, 1997; Wolf, 1998; $\mathrm{Lu}$ and Wolf, 1999) highlights that impaired GluR1mediated signaling in NAc shell may be linked to the potentiation of locomotor responding to systemic AMPH in female animals born to PolyI:C-exposed mothers. In contrast, impaired prefrontal D1R and D2R functions may contribute to the potentiation of AMPH sensitivity in male PolyI:C-offspring. Indeed, selective blockade of D1Rs in the mPFC has been shown to potentiate the locomotorenhancing effects of intra-accumbal AMPH administration in male rats (Vezina et al, 1991, 1994). Furthermore, stimulation of $\mathrm{D} 2 \mathrm{R}$ in the $\mathrm{mPFC}$ inhibits glutamatemediated excitatory drive on ventral tegmental area (VTA) dopamine neurons, thereby inhibiting mesolimbic dopaminergic activity (Harte and O'Connor, 2004).
Reduced D2R-mediated signaling in the mPFC is, thus, also expected to enhance the locomotor-stimulating effects of systemic AMPH due to impaired inhibition of mesolimbic dopaminergic activity.

Alternatively, the sex-independent pharmacological effects of prenatal immune activation may be linked to the sex-independent effects of prenatal PolyI:C exposure on TH-IR in the NAc shell (Figure 5). TH is the rate-limiting enzyme of dopamine/noradrenalin synthesis in vivo, and a positive correlation exists between the $\mathrm{TH}$ activity and dopamine synthesis in the striatum (Bacopoulos and Bhatnagar, 1977). Moreover, the locomotor enhancing effects of low doses of systemic AMPH ( $\sim 1 \mathrm{mg} / \mathrm{kg}$ for rats, $\sim 2 \mathrm{mg} / \mathrm{kg}$ for mice) have been attributed to increased dopamine transmission in the NAc (Creese and Iversen, 1975; Pijnenburg et al, 1976), especially in its shell subregion (Heidbreder and Feldon, 1998). Although we did not explicitly assess dopamine levels here, previous studies in both rats and mice have provided direct evidence that the maternal exposure to PolyI:C during pregnancy increases striatal dopamine release and/or contents in the adult offspring (Zuckerman et al, 2003; Ozawa et al, 2006). Hence, the long-term effects of prenatal PolyI:C exposure on postnatal AMPH sensitivity may readily be associated with dopaminergic imbalances especially in NAc shell, and this association may be critical for both male and female PolyI:C-offspring. Similar mechanisms may also be operational in the potentiation of AMPH sensitivity in adult rats prenatally exposed to the bacterial endotoxin lipopolysaccharide (LPS) (Fortier et al, 2004a), because maternal exposure to LPS during pregnancy has been shown to increase TH-IR in the adult offspring's nucleus accumbens (Borrell et al, 2002). Interestingly, this effect of prenatal LPS treatment was restricted to the shell but not the core subregion of nucleus accumbens (Borrell et al, 2002), which is consistent with our findings after prenatal PolyI:C exposure in mice (Figure 5). The mechanisms as to why prenatal immune activation is effective in affecting TH-IR in one but not the other subregion of nucleus accumbens essentially remain unknown thus far. Considering the distinct roles of NAc core and shell subregions in the regulation and modulation of a variety of cognitive, attentional, emotional, and reward-related behaviors (Spanagel and Weiss, 1999; Nieoullon, 2002; Weiner, 2003), further studies are clearly warranted to identify the critical mechanisms underlying the distinct effects of prenatal cytokine-associated events on dopaminergic transmission in NAc core and shell.

Since noncompetitive NMDA-receptor antagonists such as MK-801 increase dopamine release in the NAc (Mathe et al, 1996; Yan et al, 1997; Kretschmer, 1999) and stimulate the activity of VTA neurons (French et al, 1993; Murase et al, 1993), the efficacy of MK-801 to increase locomotor activity has also been attributed to increased dopaminergic transmission in mesolimbic structures. In contrast, MK801-induced hyperlocomotor activity can also occur in the absence of endogenous dopamine (Carlsson and Carlsson, 1989; Chartoff et al, 2005). It has therefore been suggested that blockade of NMDA receptors may lead to inhibition of the corticostriatal glutamatergic pathway and eventually result in excessive activation of cortical and subcortical structures, thereby stimulating locomotor activity (Carlsson 
et al, 2001). However, the different views on the neuronal substrates underlying MK-801-induced stimulation of locomotor activity are not mutually exclusive (Chartoff et al, 2005). Our immunohistochemical analyses of the NMDA-receptor subunit NR1 did not reveal any significant changes as a function of the prenatal and postnatal manipulations (Table 3). NR1 is ubiquitously expressed in the adult mammalian brain throughout pre- and postnatal development (Herlenius and Lagercrantz, 2004; Lujan et al, 2005), and it is a prerequisite to form functional NMDA receptors together with the other known subunits NR2 and NR3 (Cull-Candy et al, 2001; Kohr, 2006). Hence, we do not find any evidence for the possibility that the potentiation of locomotor responding to systemic MK-801 treatment in prenatally PolyI:C-exposed offspring is linked to altered NMDA receptor-mediated signaling at the levels of the $\mathrm{mPFC}, \mathrm{dCPu}$, and NAc. The pre- and postnatal manipulations also did not significantly affect NR1-IR in other brain regions, such as the hippocampus and thalamus (Nyffeler, Meyer, Knuesel, Yee, Feldon: unpublished observation). One possibility would therefore be that the apparent changes in dopamine-related neurochemical markers may not only account for the potentiation of AMPH sensitivity in prenatally immune-challenged offspring, but they may also contribute to the enhancement of the locomotor-stimulating effects of systemic MK-801 in these animals.

Here, we also show a potentiation of the locomotorstimulating effects of systemic AMPH in prenatal control animals that were adopted by PolyI:C-exposed surrogate mothers (Figure 3). However, the long-term consequences of prenatal immune activation and being raised by immunechallenged surrogate mothers on AMPH sensitivity appear to differ in several aspects. First, the postnatal manipulation exclusively increased locomotor responding to systemic AMPH treatment in adulthood, but not in pre-adolescence. This readily indicates that, in contrast to the effects of prenatal PolyI:C exposure in early/mid gestation, the potentiation of AMPH sensitivity in prenatal control animals that were raised by immune-challenged surrogate mothers are dependent on post-pubertal maturational processes. Second, enhanced AMPH sensitivity in prenatal control animals that were raised by immune-challenged surrogate mothers was not accompanied by any changes of dopamine-related markers at the neuroanatomical level. Instead, the adoption by immune-challenged surrogate mother increased GluR1-IR in the dCPu of prenatal control animals (Figure 8). Interestingly, this effect of the crossfostering procedure was not evident in prenatally PolyI:Ctreated offspring, that is, it did not represent a 'pure' postnatal effect. This readily indicates that putative changes of postpartum maternal factors resulting from immunological stress during pregnancy differentially affected postnatal brain and behavioral development depending on the exact prenatal treatment history of the offspring (see also Meyer et al, 2006c). Hence, it is unlikely that adoptioninduced changes in physiological parameters, such as body weight, which largely emerge independently of the prenatal treatment histories (see Meyer et al, 2006c), may account for the distinct long-term effects of the pre- and postnatal manipulations.

It remains unclear, however, to what extent the changes of GluR1-IR in the dCPu of prenatal control offspring that were raised by immune-challenged surrogate mothers may be related to enhanced locomotor responding to systemic AMPH treatment. Furthermore, the mechanisms underlying the association between the adoption by immune-challenged surrogate mothers and the emergence of brain and behavioral dysfunction in the fostered offspring are also virtually unknown to date. However, the induction of maternal stress responses might represent one way by which postnatal mother-infant interactions could be affected by immunological stimulation during pregnancy (Meek et al, 2001; Haddad et al, 2002; Patin et al, 2002; Webster and Sternberg 2004). There is considerable experimental evidence supporting the hypothesis that interference to normal postnatal motherinfant interactions can induce a variety of behavioral and pharmacological abnormalities in the grown animals (Cirulli et al, 2003; Pryce and Feldon 2003), including increased sensitivity to dopamine-related drugs (Brake et al, 2004). One alternative (but not mutually exclusive) possibility would be that PolyI:C-induced immunological stress during pregnancy may affect the quantity and/or quality of milk production, which is essential for successful postnatal development. On the other hand, it seems unlikely that prenatal PolyI:C exposure could have led to persistent inflammation in the maternal host and cytokines in the milk, because a single administration of PolyI:C is known to result a time-limited elevation of cytokines in the host (Kimura et al, 1994; Fortier et al, 2004b; Meyer et al, 2006b).

In conclusion, dopamine- and glutamate-related pharmacological and neuroanatomical dysfunctions after prenatal immune challenge in early/mid pregnancy are most likely mediated by prenatal but not postnatal maternal effects on the offspring. Since the long-term structural and functional consequences of prenatal immune activation reported here and in previous studies readily capture some critical behavioral, pharmacological, and neuropathological abnormalities implicated in schizophrenia (reviewed in Meyer et al, 2005, 2007; Nawa and Takei, 2006; Sullivan et al, 2006), our findings support the hypothesis that inflammation-mediated disruption of early prenatal brain development may predispose the organism for the emergence of schizophrenia-related psychopathology in adulthood. This should thus further encourage attempts to develop early preventive strategies to reduce the risk for postnatal psychopathology associated with disturbances in early brain development after in utero immune challenge. Furthermore, since prenatal immune activation in early/mid pregnancy may lead to dopamine-associated endogenous sensitization mechanisms before the onset of the full spectrum of psychopathology, early prenatal PolyI:C exposure in mice may be applied as an experimental tool for the longitudinal monitoring of neurochemical abnormalities suggested to be involved in the etiopathology and disease process of schizophrenia (Laruelle, 2000). The present study has also further highlighted that being reared by surrogate mothers that experienced immunological stress during pregnancy as such may constitute a risk factor for specific behavioral and pharmacological abnormalities in adult life, a finding that is of clear relevance to developmental psychobiology in general. 


\section{ACKNOWLEDGEMENTS}

The present study was supported by SNF Grant No. 3100AO-100309 and Grants of the Swiss Federal Institute of Technology No. TH-9/04-2 and TH-22/04-1. We are extremely grateful to Liz Weber, Peter Schmid, and Hannes Sigrist for their technical support, to Natalie AeschbachJones for her editorial assistance, and to the Animal Services Department, Schwerzenbach for animal husbandry and care.

\section{DISCLOSURE}

The authors have no conflicts to disclose.

\section{REFERENCES}

Bacopoulos NG, Bhatnagar RK (1977). Correlation between tyrosine hydroxylase activity and catecholamine concentration or turnover in brain regions. J Neurochem 29: 639-643.

Borrell J, Vela JM, Arévalo-Martin A, Molina-Holgado E, Guaza C (2002). Prenatal immune challenge disrupts sensorimotor gating in adult rats: implications for the etiopathogenesis of schizophrenia. Neuropsychopharmacology 26: 204-221.

Brake WG, Zhang TY, Diorio J, Meaney MJ, Gratton A (2004). Influence of early postnatal rearing conditions on mesocorticolimbic dopamine and behavioural responses to psychostimulants and stressors in adult rats. Eur J Neurosci 19: 1863-1874.

Brown AS, Hooton J, Schaefer CA, Zhang H, Petkova E, Babulas V et al (2004). Elevated maternal interleukin-8 levels and risk of schizophrenia in adult offspring. Am J Psychiatry 161: 889-895.

Brown AS, Susser ES (2002). In utero infection and adult schizophrenia. Ment Retard Dev Disabil Res Rev 8: 51-57.

Buka SL, Tsuang MT, Torrey EF, Klebanoff MA, Wagner RL, Yolken RH (2001). Maternal cytokine levels during pregnancy and adult psychosis. Brain Behav Immun 15: 411-420.

Carlsson A, Waters N, Holm-Waters S, Tedroff J, Nilsson M, Carlsson ML (2001). Interactions between monoamines, glutamate, and GABA in schizophrenia: new evidence. Annu Rev Pharmacol Toxicol 41: 237-260.

Carlsson M, Carlsson A (1989). The NMDA antagonist MK-801 causes marked locomotor stimulation in monoamine-depleted mice. J Neural Transm 75: 221-226.

Chartoff EH, Heusner CL, Palmiter RD (2005). Dopamine is not required for the hyperlocomotor response to NMDA receptor antagonists. Neuropsychopharmacology 30: 1324-1333.

Cirulli F, Berry A, Alleva E (2003). Early disruption of the motherinfant relationship: effects on brain plasticity and implications for psychopathology. Neurosci Biobehav Rev 27: 73-82.

Creese I, Iversen SD (1975). The pharmacological and anatomical substrates of the amphetamine response in the rat. Brain Res 83: 419-436.

Cull-Candy S, Brickley S, Farrant M (2001). NMDA receptor subunits: diversity, development and disease. Curr Opin Neurobiol 11: 327-335.

Curfs JH, Meis JF, Hoogkamp-Korstanje JA (1997). A primer on cytokines: sources, receptors, effects, and inducers. Clin Microbiol Rev 10: 742-780.

Fatemi SH (2005). Neuropsychiatric Disorders and Infection. Taylor \& Francis: London.

Fortier ME, Joober R, Luheshi GN, Boksa P (2004a). Maternal exposure to bacterial endotoxin during pregnancy enhances amphetamine-induced locomotion and startle responses in adult rat offspring. J Psychiatr Res 38: 335-345.

Fortier ME, Kent S, Ashdown H, Poole S, Boksa P, Luheshi GN (2004b). The viral mimic, polyinosinic:polycytidylic acid, induces fever in rats via an interleukin-1-dependent mechanism. Am J Physiol Regul Integr Comp Physiol 287: R759-R766.

French ED, Mura A, Wang T (1993). MK-801, phencyclidine (PCP), and PCP-like drugs increase burst firing in rat A10 dopamine neurons: comparison to competitive NMDA antagonists. Synapse 13: 108-116.

Fritschy JM, Weinmann O, Wenzel A, Benke D (1998). Synapsespecific localization of NMDA and GABA(A) receptor subunits revealed by antigen-retrieval immunohistochemistry. J Comp Neurol 390: 194-210.

Gilmore JH, Jarskog LF (1997). Exposure to infection and brain development: cytokines in the pathogenesis of schizophrenia. Schizophr Res 24: 365-367.

Gilmore JH, Jarskog LF, Vadlamudi S, Lauder JM (2004). Prenatal infection and risk for schizophrenia: IL-1beta, IL-6, and TNFalpha inhibit cortical neuron dendrite development. Neuropsychopharmacology 29: 1221-1229.

Haddad JJ, Saade NE, Safieh-Garabedian B (2002). Cytokines and neuro-immune-endocrine interactions: a role for the hypothalamic-pituitary-adrenal revolving axis. J Neuroimmunol 133: $1-19$.

Harte M, O'Connor WT (2004). Evidence for a differential medial prefrontal dopamine D1 and D2 receptor regulation of local and ventral tegmental glutamate and GABA release: a dual probe microdialysis study in the awake rat. Brain Res 1017: 120-129.

Heidbreder C, Feldon J (1998). Amphetamine-induced neurochemical and locomotor responses are expressed differentially across the anteroposterior axis of the core and shell subterritories of the nucleus accumbens. Synapse 29: 310-322.

Herlenius E, Lagercrantz H (2004). Development of neurotransmitter systems during critical periods. Exp Neurol 190: S8-S21.

Holcomb HH, Lahti AC, Medoff DR, Cullen T, Tamminga CA (2005). Effects of noncompetitive NMDA receptor blockade on anterior cingulate cerebral blood flow in volunteers with schizophrenia. Neuropsychopharmacology 30: 2275-2282.

Jarskog LF, Xiao H, Wilkie MB, Lauder JM, Gilmore JH (1997). Cytokine regulation of embryonic rat dopamine and serotonin neuronal survival in vitro. Int J Dev Neurosci 15: 711-776.

Javitt DC, Zukin SR (1991). Recent advances in the phencyclidine model of schizophrenia. Am J Psychiatry 148: 1301-1308.

Kimura M, Toth LA, Agostini H, Cady AB, Majde JA, Krueger JM (1994). Comparison of acute phase responses induced in rabbits by lipopolysaccharide and double-stranded RNA. Am J Physiol 267: R1596-R1605.

Kohr G (2006). NMDA receptor function: subunit composition versus spatial distribution. Cell Tissue Res 326: 439-446.

Kretschmer BD (1999). Modulation of the mesolimbic dopamine system by glutamate: role of NMDA receptors. J Neurochem 73: 839-848.

Krystal JH, Karper LP, Seibyl JP, Freeman GK, Delaney R, Bremner JD et al (1994). Subanesthetic effects of the noncompetitive NMDA antagonist, ketamine, in humans. Psychotomimetic, perceptual, cognitive, and neuroendocrine responses. Arch Gen Psychiatry 51: 199-214.

Lahti AC, Koffel B, LaPorte D, Tamminga CA (1995). Subanesthetic doses of ketamine stimulate psychosis in schizophrenia. Neuropsychopharmacology 13: 9-19.

Laruelle M (2000). The role of endogenous sensitization in the pathophysiology of schizophrenia: implications from recent brain imaging studies. Brain Res Rev 31: 371-384.

Laruelle M, Abi-Dargham A, van Dyck CH, Gil R, D’Souza CD, Erdos J et al (1996). Single photon emission computerized tomography imaging of amphetamine-induced dopamine release in drug-free schizophrenic subjects. Proc Natl Acad Sci USA 93: 9235-9240.

Lieberman JA, Kane JM, Alvir J (1987). Provocative tests with psychostimulant drugs in schizophrenia. Psychopharmacology 91: 415-433. 
Ling ZD, Potter ED, Lipton JW, Carvey PM (1998). Differentiation of mesencephalic progenitor cells into dopaminergic neurons by cytokines. Exp Neurol 149: 411-423.

Lu W, Chen H, Xue CJ, Wolf ME (1997). Repeated amphetamine administration alters the expression of mRNA for AMPA receptor subunits in rat nucleus accumbens and prefrontal cortex. Synapse 26: 269-280.

Lu W, Wolf ME (1999). Repeated amphetamine administration alters AMPA receptor subunit expression in rat nucleus accumbens and medial prefrontal cortex. Synapse 32: 119-131.

Lujan R, Shigemoto R, Lopez-Bendito G (2005). Glutamate and GABA receptor signalling in the developing brain. Neuroscience 130: $567-580$.

Mathe JM, Nomikos GG, Hildebrand BE, Hertel P, Svensson TH (1996). Prazosin inhibits MK-801-induced hyperlocomotion and dopamine release in the nucleus accumbens. Eur J Pharmacol 309: $1-11$.

Meek LR, Dittel PL, Sheehan MC, Chan JY, Kjolhaug SR (2001). Effects of stress during pregnancy on maternal behavior in mice. Physiol Behav 72: 473-479.

Meyer U, Feldon J, Schedlowski M, Yee BK (2005). Towards an immuno-precipitated neurodevelopmental animal model of schizophrenia. Neurosci Biobehav Rev 29: 913-947.

Meyer U, Feldon J, Schedlowski M, Yee BK (2006a). Immunological stress at the maternal-foetal interface: a link between neurodevelopment and adult psychopathology. Brain Behav Immun 20: $378-388$.

Meyer U, Nyffeler M, Engler A, Urwyler A, Schedlowski M, Knuesel I et al (2006b). The time of prenatal immune challenge determines the specificity of inflammation-mediated brain and behavioral pathology. J Neurosci 26: 4752-4762.

Meyer U, Schwendener S, Feldon J, Yee BK (2006c). Prenatal and postnatal maternal contributions in the infection model of schizophrenia. Exp Brain Res 173: 243-257.

Meyer U, Yee BK, Feldon J (2007). The neurodevelopmental impact of prenatal infections at different times of pregnancy: the earlier the worse? Neuroscientist (in press).

Miller MT, Stromland K, Ventura L, Johansson M, Bandim JM, Gillberg C (2005). Autism associated with conditions characterized by developmental errors in early embryogenesis: a mini review. Int J Dev Neurosci 23: 201-219.

Murase S, Mathe JM, Grenhoff J, Svensson TH (1993). Effects of dizocilpine (MK-801) on rat midbrain dopamine cell activity: differential actions on firing pattern related to anatomical localization. J Neural Transm Gen Sect 91: 13-25.

Nawa H, Takei N (2006). Recent progress in animal modeling of immune inflammatory processes in schizophrenia: implication of specific cytokines. Neurosci Res 56: 2-13.

Nieoullon A (2002). Dopamine and the regulation of cognition and attention. Prog Neurobiol 67: 53-83.

Ozawa K, Hashimoto K, Kishimoto T, Shimizu E, Ishikura H, Iyo M (2006). Immune activation during pregnancy in mice leads to dopaminergic hyperfunction and cognitive impairment in the offspring: a neurodevelopmental animal model of schizophrenia. Biol Psychiatry 59: 546-554.

Patin V, Lordi B, Vincent A, Thoumas JL, Vaudry H, Caston J (2002). Effects of prenatal stress on maternal behavior in the rat. Dev Brain Res 139: 1-8.

Peleg-Raibstein D, Sydekum E, Russig H, Feldon J (2006). Withdrawal from continuous amphetamine administration abolishes latent inhibition but leaves prepulse inhibition intact. Psychopharmacology 185: 226-239.

Peterson JD, Wolf ME, White FJ (2006). Repeated amphetamine administration decreases D1 dopamine receptor-mediated inhibition of voltage-gated sodium currents in the prefrontal cortex. J Neurosci 26: 3164-3168.

Pijnenburg AJ, Honig WM, Van der Heyden JA, Van Rossum JM (1976). Effects of chemical stimulation of the mesolimbic dopamine system upon locomotor activity. Eur J Pharmacol 35: 45-58.

Pryce CR, Feldon J (2003). Long-term neurobehavioural impact of the postnatal environment in rats: manipulations, effects and mediating mechanisms. Neurosci Biobehav Rev 27: 57-71.

Rasier G, Toppari J, Parent AS, Bourguignon JP (2006). Female sexual maturation and reproduction after prepubertal exposure to estrogens and endocrine disrupting chemicals: a review of rodent and human data. Mol Cell Endocrinol 254-255: 187-201.

Rodier PM, Hyman SL (1998). Early environmental factors in autism. Men Retard Dev Disabil Res Rev 4: 121-128.

Russig H, Murphy CA, Feldon J (2005). Behavioural consequences of withdrawal from three different administration schedules of amphetamine. Behav Brain Res 165: 26-35.

Shi L, Fatemi SH, Sidwell RW, Patterson PH (2003). Maternal influenza infection causes marked behavioral and pharmacological changes in the offspring. J Neurosci 23: 297-302.

Sisk CL, Foster DL (2004). The neural basis of puberty and adolescence. Nat Neurosci 7: 1040-1047.

Spanagel R, Weiss F (1999). The dopamine hypothesis of reward: past and current status. Trends Neurosci 22: 521-527.

Spear LP (2000). The adolescent brain and age-related behavioral manifestations. Neurosci Biobehav Rev 24: 417-463.

Sullivan R, Wilson DA, Feldon J, Yee BK, Meyer U, Richter-Levin G et al (2006). The international society for developmental psychobiology annual meeting symposium: impact of early life experiences on brain and behavioral development. Dev Psychobiol 48: 583-602.

Tzschentke TM (2001). Pharmacology and behavioral pharmacology of the mesocortical dopamine system. Prog Neurobiol 63: 241-320.

Vezina P, Blanc G, Glowinski J, Tassin JP (1991). Opposed behavioural outputs of increased dopamine transmission in prefrontocortical and subcortical areas: a role for the cortical D-1 dopamine receptor. Eur J Neurosci 3: 1001-1007.

Vezina P, Blanc G, Glowinski J, Tassin JP (1994). Blockade of D-1 dopamine receptors in the medial prefrontal cortex produces delayed effects on pre- and postsynaptic indices of dopamine function in the nucleus accumbens. Synapse 16: 104-112.

Webster JI, Sternberg EM (2004). Role of the hypothalamicpituitary-adrenal axis, glucocorticoids and glucocorticoid receptors in toxic sequelae of exposure to bacterial and viral products. J Endocrinol 181: 207-221.

Weiner I (2003). The 'two-headed' latent inhibition model of schizophrenia: modeling positive and negative symptoms and their treatment. Psychopharmacology 169: 257-297.

Wolf ME (1998). The role of excitatory amino acids in behavioral sensitization to psychomotor stimulants. Prog Neurobiol 54: 679-720.

Wu J, Zou H, Strong JA, Yu J, Zhou X, Xie Q et al (2005). Bimodal effects of MK-801 on locomotion and stereotypy in C57BL/6 mice. Psychopharmacology 177: 256-263.

Yan QS, Reith ME, Jobe PC, Dailey JW (1997). Dizocilpine (MK801) increases not only dopamine but also serotonin and norepinephrine transmissions in the nucleus accumbens as measured by microdialysis in freely moving rats. Brain Res 765: 149-158.

Zuckerman L, Rehavi M, Nachman R, Weiner I (2003). Immune activation during pregnancy in rats leads to a postpubertal emergence of disrupted latent inhibition, dopaminergic hyperfunction, and altered limbic morphology in the offspring: a novel neurodevelopmental model of schizophrenia. Neuropsychopharmacology 28: 1778-1789.

Zuckerman L, Weiner I (2005). Maternal immune activation leads to behavioral and pharmacological changes in the adult offspring. J Psychiatr Res 39: 311-323. 